\title{
Deregulation of HLA-I in cancer and its central importance for immunotherapy
}

\author{
Ahmet Hazini, Kerry Fisher, Len Seymour
}

To cite: Hazini A, Fisher K, Seymour L. Deregulation of HLA-I in cancer and its central importance for immunotherapy. Journal for ImmunoTherapy of Cancer 2021;9:e002899. doi:10.1136/jitc-2021-002899

Accepted 21 June 2021

\section{Check for updates}

(C) Author(s) (or their employer(s)) 2021. Re-use permitted under CC BY-NC. No commercial re-use. See rights and permissions. Published by BMJ.

Department of Oncology, University of Oxford, Oxford, Oxfordshire, UK

Correspondence to

Dr Len Seymour;

len.seymour@oncology.ox.ac.uk

\begin{abstract}
It is now well accepted that many tumors undergo a process of clonal selection which means that tumor antigens arising at various stages of tumor progression are likely to be represented in just a subset of tumor cells. This process is thought to be driven by constant immunosurveillance which applies selective pressure by eliminating tumor cells expressing antigens that are recognized by $T$ cells. It is becoming increasingly clear that the same selective pressure may also select for tumor cells that evade immune detection by acquiring deficiencies in their human leucocyte antigen (HLA) presentation pathways, allowing important tumor antigens to persist within cells undetected by the immune system. Deficiencies in antigen presentation pathway can arise by a variety of mechanisms, including genetic and epigenetic changes, and functional antigen presentation is a hard phenomenon to assess using our standard analytical techniques. Nevertheless, it is likely to have profound clinical significance and could well define whether an individual patient will respond to a particular type of therapy or not. In this review we consider the mechanisms by which HLA function may be lost in clinical disease, we assess the implications for current immunotherapy approaches using checkpoint inhibitors and examine the prognostic impact of HLA loss demonstrated in clinical trials so far. Finally, we propose strategies that might be explored for possible patient stratification.
\end{abstract}

\section{INTRODUCTION}

The major histocompatibility complex class I (MHC-I) proteins in humans are termed as human leucocyte antigen I (HLA-I) and they are divided into classical and non-classical HLA-I subtypes. ${ }^{1}$ Classical type I HLA molecules (HLA-A, HLA-B, and HLA-C) function to present cellular antigens to $\mathrm{T}$ cells and are essential for immunosurveillance and cancer immunotherapy. It is now clear that loss of HLA-I function is an important escape mechanism for tumors from immunotherapy, by a variety of mechanisms ${ }^{2-4}$ Between $60 \%$ and $90 \%$ of patients may be affected even before treatment, and this would render them unable to respond to all current immunotherapy approaches. ${ }^{5}$ On the other hand, a growing number of studies suggest that nonclassical HLA-I molecules also play a critical role in cancer immune escape.

\section{Classical HLA class I expression}

Classical HLA-I molecules, which function to present cellular antigens to T cells, consist of a highly polymorphic $\alpha$-heavy chain encoded by HLA-A, HLA-B or HLA-C genes and $\beta-2-$ microglobulin $(\beta 2 \mathrm{M})$ light chain which provides stability to the HLA-I complex. ${ }^{6}$ Each HLA molecule has a cleft that binds antigen-derived peptides and presents them to T cells. Several cellular components are involved in the antigen processing and presentation mechanism. Following ubiquitinylation intracellular proteins are degraded by the immunoproteasomes to produce short peptides for transportation by the transporter associated with antigen processing (TAP1/ TAP2) into the endoplasmic reticulum (ER). The glycoprotein Tapasin, in cooperation with other chaperones (calreticulin, ERp57) mediates an interaction between TAP $1 / 2$ and newly synthesized HLA-I molecules, forming peptide/HLA-I complexes which are transported to the cell surface for T cell surveillance and possible antigen-specific recognition (figure 1). Classical HLA-I molecules can also be recognized by killer cell immunoglobulinlike receptors (KIRs) expressed on natural killer (NK) cells and inhibit their HLA-I independent cytotoxic function. ${ }^{7}$

\section{Non-classical HLA class I expression}

In contrast to classical HLA-I molecules, non-classical HLA-E, HLA-F and HLA-G have immunosuppressive functions. HLA-E consists of a heavy chain, $\beta 2 \mathrm{M}$ and a bound peptide derived from leader peptide sequences of other HLA class I molecules. Its main function appears to be allowing NK cells indirectly to monitor expression of the other class I HLA molecules and prevent NK attack while simultaneously suppressing $\mathrm{T}$ cell cytotoxicity. ${ }^{8}$ It can be expressed in all nucleated cells at low basal levels, although it is often abundant on trophoblast and tumor cells. ${ }^{9}$ HLA-F and HLA-G were also originally identified as expressed on trophoblastic cells to provide fetal-maternal immune tolerance. ${ }^{1011}$ HLA-F has structural similarities to HLA-E 


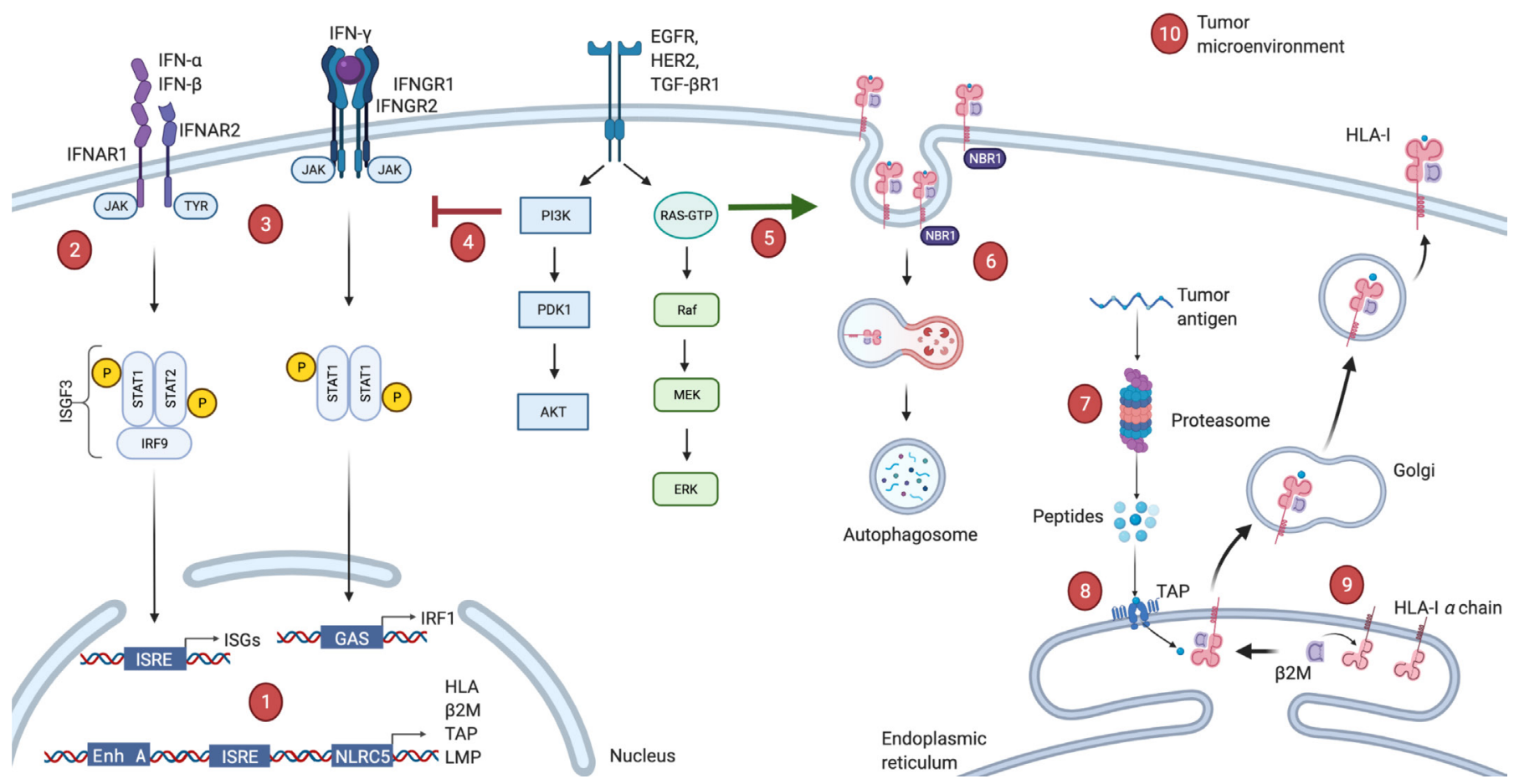

Figure 1 Possible deficiencies in antigen presenting machinery. (1) Acquired mutations, transcriptional or post-transcriptional regulations in HLA or antigen presentation machinery (APM) genes or epigenetic modifications in their promoter regions. (2, 3) Defects in type-I or type-II interferon pathways, which are direct stimulators of HLA-I expression. (4) Aberrant activation of PI3K-Akt oncogenic pathway interferes with phosphorylation of STAT1 and hinders interferon mediated HLA-I expression. (5) Oncogenic BRAF mutation can drive internalization and endosomal degradation of surface HLA-I antigens. (6) Autophagy cargo receptor NBR1 protein can bind to HLA-I leads to autophagy-mediated degradation. (7) Defects in the proteasome components (LMP2, LMP9 or MECL-1, etc). (8) Defects in the peptide transport or ER peptide loading complex (TAP1/TAP2, ERp57, calnexin, calreticulin). (9) Downregulation of light chain $\beta 2 \mathrm{M}$ can lead to complete absence of HLA-I. (10) Microenvironmental conditions such as glucose deprivation, hypoxia, acidosis or excessive IL-10, TGF- $\beta$ levels can also drive loss of HLA. $\beta 2 \mathrm{M}$, $\beta$-2-microglobulin; ER, endoplasmic reticulum; HLA, human leucocyte antigen; IFNAR1, interferon alpha and beta receptor 1; IL-10, interleukin 10; ISRE, interferon stimulated response element; TAP1, transporter associated with antigen processing 1; TGF- $\beta$, transforming growth factor- $\beta$.

and classical HLA-I antigens although its complete function remains to be elucidated, as it is also expressed on activated immune cells. ${ }^{12}$ Unlike other HLA-I antigens, HLA-G comprises four membrane-bound and three soluble isoforms (sHLA-G) as a result of alternative RNA splicing. Except HLA-G1 and HLA-G5, the other five HLA-G isoforms do not associate with $\beta 2 \mathrm{M} .{ }^{13}$ The isoform variations and structural complexity make precise HLA-G measurements particularly challenging. ${ }^{14}$ HLA-G is known to interact with all the main immune cell subsets and can inhibit their cytotoxicity via various receptors (Expression of HLA-G). ${ }^{15}$

\section{WHAT HAPPENS TO HLA-I IN CANCER?}

The multistep nature of the antigen-presentation process provides transformed cells with a variety of options to deregulate antigen presentation at genetic, epigenetic, transcriptional or post-transcriptional levels, and thereby to evade immune surveillance (figure 1$)^{16}$. In this section, we outline some of the known cancer-associated changes and assess their frequency of occurrence. Tumor heterogeneity means that a range of changes can occur within a single tumor ${ }^{17}$, making it hard to state frequencies accurately, nevertheless it is possible to get some insight into the patterns of HLA loss that occur in different cancer types. The types of defects which lead to surface HLA-I aberration can be divided into two main groups: irreversible or reversible (sometimes also referred as hard or soft), reflecting whether restoration of HLA-I expression is possible following cytokine or pharmaceutical treatment. $^{18}$

\section{Irreversible HLA defects}

Molecular changes to the coding regions of HLA or antigen presentation machinery (APM) component genes are often described as 'irreversible' defects. They are identified empirically by searching for mutations in HLA class I molecules using DNA sequencing. Recovery of HLA-I expression can be only possible when these mutated genes are compensated with intact ones by gene editing or delivery techniques.

\section{HLA-I gene mutations}

The most commonly observed genetic change is loss of heterozygosity (LOH) of the $H L A-A B C$ genes located at 
A

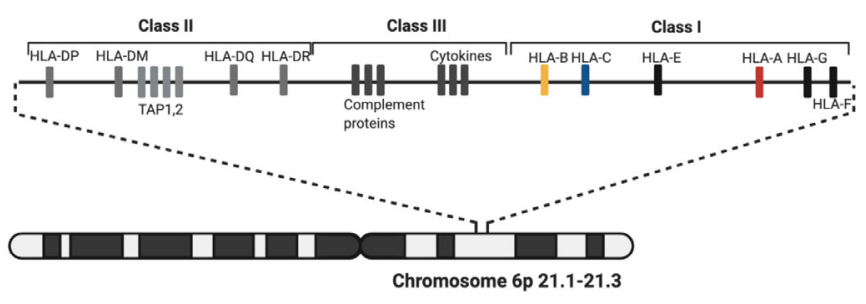

B
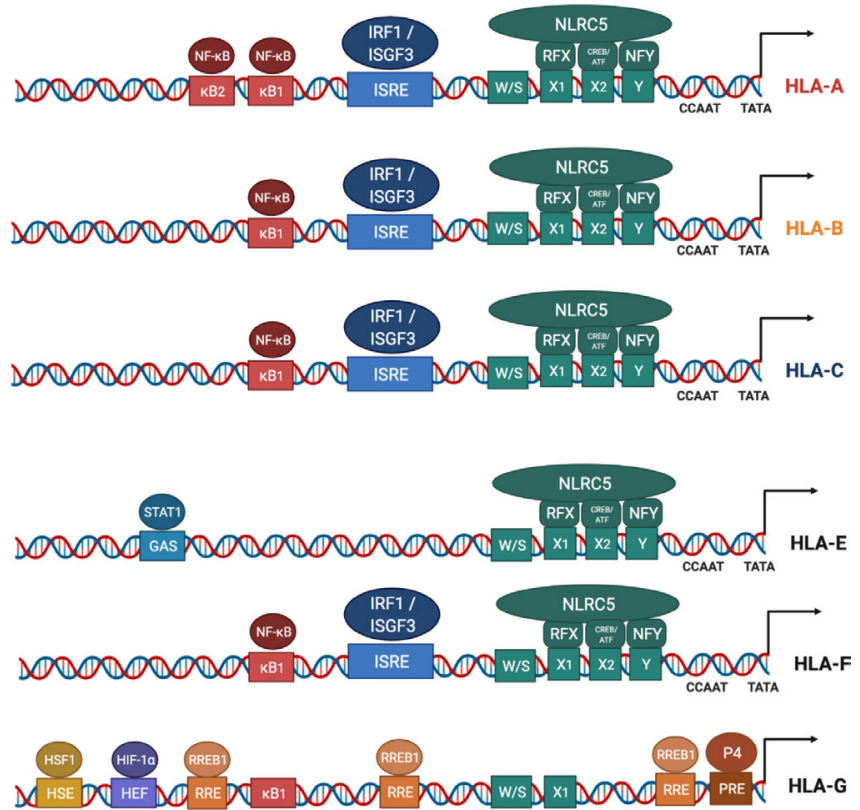

Figure 2 Schematic overview of genomic localization of HLA molecules and cisregulatory elements in promoter region of classical and non-classical HLAs. (A) Genes encoding HLAs and APM proteins are localized on the chromosome 6 short arm. (B) Classical HLA genes promoters comprise two major regulatory modules; NF- $\mathrm{KB}$ response element and ISRE consisting upstream nodule and SXY enhanceosome nodule. TATA and CCAAT elements controlling basal transcription of these genes. Non classical HLA-E and HLA-G promoter regions have some differences. HLA-E gene can be transactivated by NLRC5 and IFN- $\gamma$ but not NF-KB. HLA-F regulatory nodules shows high homology to classical HLA-I. Differently, HLA-G promoter region has diverse binding sites such as HSE, HEF, RRE and PRE which overlaps TATA box. HDAC1 can interact with RREB1 increases chromatin condensation. HLA-G promoter NF- $\mathrm{kB}$ binding sites can only bind p50 homodimers therefore NF- $\mathrm{KB}$ has no transactivator function. NF-KB, kappa-light-chain-enhancer of activated B cells; APM, antigen presentation machinery; HLAs, human leucocyte antigen; HSE, heat shock response element; IFN- $\gamma$, interferon- $\gamma$; NLRC5, NOD-like receptor caspase protein 5; ISRE, interferon stimulated response element; PRE, progesterone response element.

chromosome locus 6 p21 which is reported to occur at a frequency of around $40 \%-54 \%$ in non-small cell lung carcinoma (NSCLC) and $44 \%$ in colorectal cancer ${ }^{19} 20$ (figure 2A). The HLA gene is the most polymorphic gene in the human genome, essential for its role of presenting a wide range of peptides. $\mathrm{LOH}$ at chromosome 6 leads to complete loss of either parental or maternal HLA haplotypes. This means that the repertoire of peptides normally presented by that haplotype is lost, potentially preventing presentation of important neoantigens. Simultaneously maintaining expression of the HLA haplotype from the other parent staves off attack by NK cells. Perhaps the best-defined example of this arose from the TRACERx analysis of early-stage patients with lung cancer, where McGranahan et al showed that $40 \%$ of patients had HLA $\mathrm{LOH}$, using a novel computational tool determining HLAallele specific copy number. ${ }^{20}$ High levels of neoantigens predicted to bind to the lost HLA allele were identified. Due to its subclonal nature they inferred that HLA LOH was a late event in tumor evolution and occurred under selective pressure as just one mechanism for cancer cells to avoid presenting neoantigens and consequent immune elimination.

Loss of a functional HLA alleles by mutation or gene deletion is another but less frequent structural defect. A recent analysis detected non-silent genetic mutations in HLA genes in approximately $3.3 \%$ of all tumors, with greater prevalence for head and neck, lung, stomach and colorectal cancer. ${ }^{21}$ Loss of a functional HLA allele through mutation would have similar consequences as HLA LOH, precluding presentation of immunodominant peptides such as MART-1 following loss of HLA-A2 in melanoma. ${ }^{22} \mathrm{~A}$ striking example was observed in a clinical study (NCT01174121) in which patients were screened for tumor antigen profile and then treated with ex vivo expanded antigen specific autologous T cells. One patient who responded to HLA-C*08:02 restricted KRAS G12D targeted T cells therapy subsequently encountered tumor relapse. Whole exome analysis revealed loss of the chromosome 6 haplotype encoding HLA-C*08:02 in progressing tumors. ${ }^{23}$ This case report shows how cancer cells can acquire significant genetic changes when under strong selective pressure by antigen specific $\mathrm{T}$ cells.

\section{Mutations in B2M}

Another important irreversible defect is the loss of $\beta 2 \mathrm{M}$ protein expression. This generally arises by a combination of two events; LOH on chromosome 15 (15q21) and mutation in the remaining $\beta 2 M$ allele. This type of alteration results in complete loss of HLA presentation, as $\beta 2 \mathrm{M}$ is responsible for stability of the HLA-I complex. ${ }^{24}$ It has been suggested that $\mathrm{LOH}$ at chromosome 15 is an earlier event and selective pressure causes mutations in the second $\beta 2 \mathrm{M}$ copy which leads to complete loss of 
functional antigen presentation. ${ }^{25}$ Non-silent mutations in $\beta 2 \mathrm{M}$ have been reported in a broad range of patients ${ }^{26}$ and LOH at chromosome 15q21, assessed using two microsatellite markers, was found in $44 \%$ of bladder carcinomas $(n=69), 35 \%$ of colon carcinomas $(n=95), 16 \%$ of melanomas $(n=70)$ but only $7 \%$ of renal cancers $(n=45){ }^{27-29}$ Interestingly the rate of mutations was appreciably higher in microsatellite unstable disease (particularly colorectal, gastric and uterine cancers) and was associated with an increased overall burden of mutations for both microsatellite stable and unstable disease, commensurate with the role of the HLA system in presenting acquired mutations for immunosurveillance. Moreover, loss of B2M mediated acquired resistance to immune checkpoint inhibitors (ICIs) has been reported in clinical investigations. ${ }^{30-33}$

\section{Mutations in interferon pathway genes}

Type-I and type-II interferons are strong inducers of HLA-I expression and they have pro-apoptotic effects on tumor cells. ${ }^{34-36}$ Therefore, not surprisingly, they become important targets for tumors during immune evasion, disease progression and metastasis. Particularly, interferon- $\gamma(\mathrm{IFN}-\gamma)$ is master regulator of several APM genes and the most potent HLA molecule inducer. It is strongly secreted by activated $\mathrm{T}$ cells and upregulates expression of MHC class-I and MHC class-II transactivator (CIITA) proteins, NOD-like receptor caspase recruitment domain containing protein 5 (NLRC5) and MHC CIITA, respectively. ${ }^{37}$ The HLA heavy chains and APM promoters contain interferon stimulated response element (ISRE) region, the binding site of interferon regulatory factor-1 (IRF1) (figure 2), which is induced via the JAK/STAT pathway following IFN- $\gamma$ binding to the IFNGR1 extracellular domain. ${ }^{38}$ In tumor cells, loss of function mutations in $J A K / S T A T$ or IRF1 genes can remove this responsiveness to IFN- $\gamma$, and JAK/STAT pathway mutations have been reported for several cancer types. They are associated with poor prognosis of the disease and resistance to immunotherapies. ${ }^{39-41}$ Alternatively, missense mutations in the IFNGR1 gene can cause loss of cell surface IFGNR1, thus inhibiting IFN- $\gamma$ responsiveness. ${ }^{42}{ }^{43}$ In addition, IFN- $\gamma$ suppressor genes, SOC1 and PIAS4 have also been shown to be upregulated in patients who do not respond to checkpoint blockade. ${ }^{39}$

\section{Mutations in genes involved in antigen presentation}

Mutations in the more obvious HLA and associated genes may be only the tip of the iceberg. Acquired genetic mutations may also be present in other components of antigen presentation, for example in immune proteasomal degradation pathways ${ }^{44}$ (such as LMP2, LMP9, MECL-1) or the ER peptide loading complex (for example tapasin, ERp57, calnexin, calreticulin) ${ }^{45}{ }^{46}$ Hence our knowledge of genetic changes leading to dysfunctional class I antigen presentation is likely still in its infancy, and there may be myriad other genetic changes exploited by tumors that we have not so far identified.

\section{Reversible HLA defects}

Loss-of-functional HLA presentation resulting from epigenetic silencing, transcriptional or posttranscriptional/translational modifications of the HLA genes themselves or of any other gene essential for antigen processing, are often termed 'reversible' defects. In principle, HLA function might be restored in these cancers using pharmacological interventions (Figure 3).

\section{Epigenetic silencing}

Histone deacetylation and DNA methylation are epigenetic gene regulatory mechanisms which are crucial for tissue specific gene expression and homeostasis. Histone deacetylase (HDAC) and DNA methyltransferase (DNMT) act as gene silencers by condensing the chromatin structure. Cancer cells can use these mechanisms to inhibit tumor suppressor genes or apoptosis ${ }^{47}$ alongside other mechanisms that can lead to down regulation of $\beta 2 \mathrm{M}$ and other APM proteins. ${ }^{48} 49$ Type-I and type-II IFNs levels were shown to be upregulated following inhibition of these epigenetic mechanisms. ${ }^{50}$ For example, decreased STAT1 expression in squamous cell carcinoma of head and neck (SCCHN) was associated with promoter methylation, and levels could be restored in SCCHN cell lines using the DNMT inhibitor azacytidine. ${ }^{51}$ Several studies have now shown that HLA expression can sometimes be restored using HDAC or DNMT inhibitors. ${ }^{52}$ Recently, synergistic effects of the US Food and Drug Administration (FDA) approved HDAC inhibitors and checkpoint inhibitors have been reported in preclinical studies, suggesting HDAC inhibitors can restore functional HLA in some patients ${ }^{53-56}$ and additional clinical studies are underway. ${ }^{5758}$ At the data cut-off, objective response rates were reported as $31 \%, 19 \%$, and $18 \%$ in patients with uveal melanoma $(n=29)$, NSCLC $(n=57)$, and melanoma $(\mathrm{n}=53)$, respectively, in phase 2 studies. ${ }^{59-61}$ It is perhaps worth noting that Assay for Transposase Accessible Chromatin with high-throughput sequencing (ATAC-seq) has become an effective technology in cancer epigenetics research ${ }^{62}$ and it gives the opportunity to investigate small sample sizes, which is a common issue in clinical studies. Adapting such techniques to ongoing studies should provide a better understanding of the impact of epigenetic therapy on HLA-I expression.

\section{Transcriptional silencing}

Transcription of classical HLA and other antigen presentation pathway genes is controlled by two regulatory modules (figure 2). The upstream module consists of the enhancer $\mathrm{A}$ containing $\mathrm{\kappa B} 1$ and $\kappa \mathrm{B} 2$ binding sites for NF-אB family members p50, p65, and c-Rel and ISRE with binding sites for IRF1 and IRF2, together with binding sites for USF1 and USF2 and Sp1. The downstream 'SXY' module has binding sites for a multiprotein complex of RFX, CREB/ATF and NFY. It was recently reported that NLRC5 translocates to the SXY-module, forms an enhanceosome together with RFX-complex and activates HLA-I transcription. ${ }^{37} 63$ Together these two modules provide 
Genetic defects in HLA expression
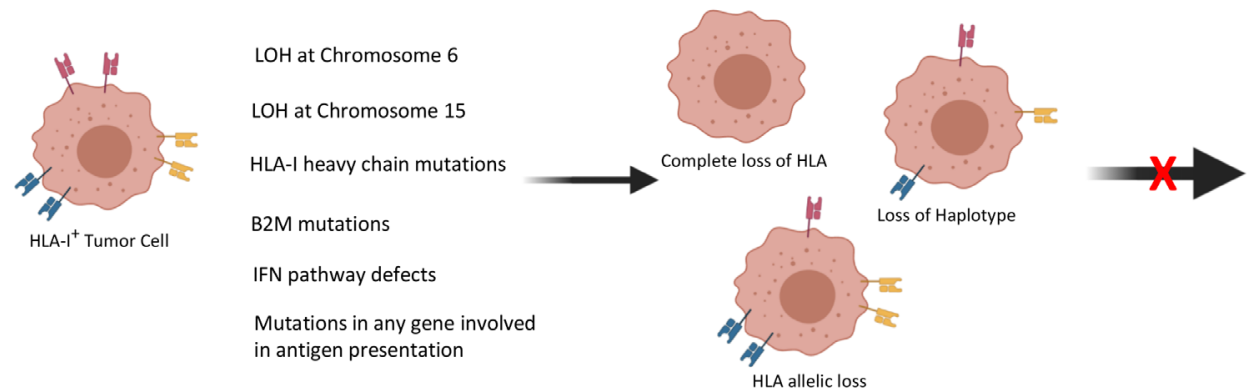

HLA-I expression not recoverable by conventional pharmacological interventions (hard mutations) in antigen presentation
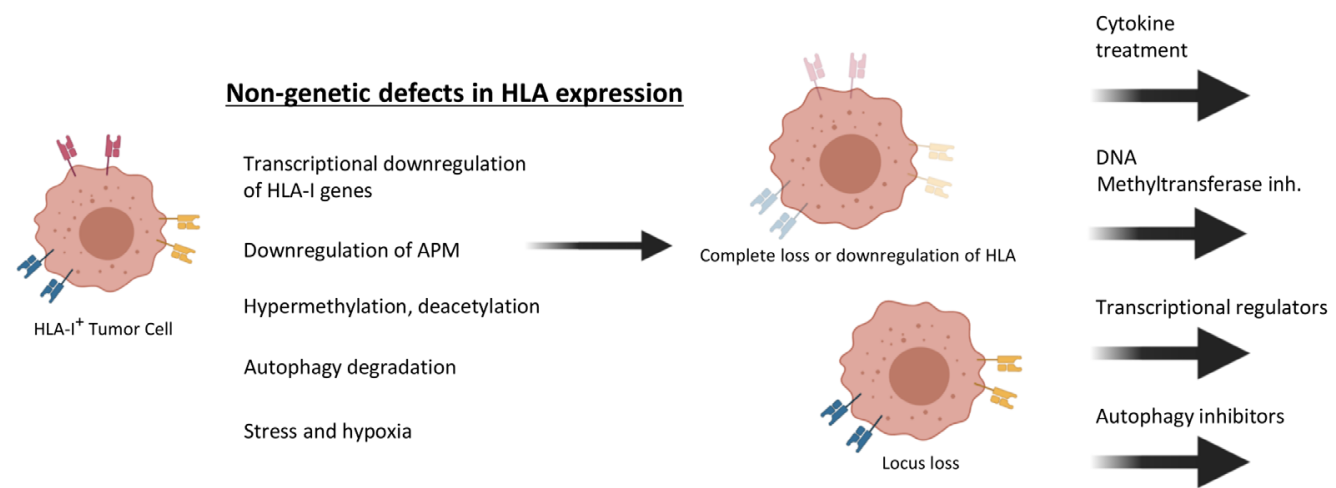

Figure 3 Type of classical HLA-I alterations in cancer cells. Various HLA-I abnormal cancer phenotypes can form depending on whether these abnormalities are caused by genetic or non-genetic defects. The striking difference between these defects is genetic aberration-originated HLA-I dysregulation cannot be recovered by pharmaceutical or IFN treatment. The most frequently formed genetic abnormality is LOH at chromosome 6 which leads to loss of maternal or paternal HLA haplotypes. Heavy chain mutations or $\beta 2 \mathrm{M}$ mutations causes complete loss of HLA function. HLA allelic loss may occur in the case of locus-specific mutations. Any mutations in IFN signaling pathway or APM genes may lead to complete loss or down regulation of HLA-I expression. On the other hand, cancer-specific transcriptional down regulation of HLA-I genes or APM genes, epigenetic changes such as methylation or acetylation, autophagy-mediated degradation, stress and hypoxia can lead to HLA-I expression dysregulation. However, HLA-I can be recovered by using different treatment approaches in accordance with the specific type of defect. APM, antigen presentation machinery; $\beta 2 \mathrm{M}, \beta$-2-microglobulin; IFN, interferon; LOH, loss of heterozygosity; HLA, human leucocyte antigen.

constitutive and regulatory control of classical HLA-I gene expression $^{64}$ (figure 2B). Due to this transactivator role of NLRC5 it is suggested to be a key target for immune evasion in many cancer types. ${ }^{65}$ Analysis in large cohort human cancer samples revealed that high NLRC5 expression is correlated with higher HLA expression and better survival. ${ }^{66}$ Another regulator of HLA class I expression is suggested to be tumor suppressor Fhit protein. ${ }^{67}$ Although its mechanism has not been clearly identified, Fhit-transfected cancer cells recovered MHC-I expression independent of NLRC5. Additionally, loss of Fhit expression in human breast tumors is associated with loss of HLA-I molecules. ${ }^{68}$

The non-classical HLA-I molecules (notably HLA-E and HLA-G) have slightly different regulatory sequences, providing the opportunity for differential expression of classical and non-classical HLA genes through transcription factor variation (figure 2B). Both regulatory modules mentioned above can be identified in the HLA-E and HLA-F promoter regions, however in HLA-E the $\kappa \mathrm{B} 1$ and $\kappa \mathrm{B} 2$ and ISRE sites are different from the classical genes meaning that HLA-E is thought to be not induced by NF- $\mathrm{KB}$, although an upstream GAS site provides strong inducibility by IFN- $\gamma$. HLA-E shows some induction by binding of NLRC5 to the SXY domain. The HLA-F promoter structure exhibits strong homology to classical HLA-I antigens, consisting of one NF- $\mathrm{KB}$ binding site followed by ISRE and SXY nodule. On the other hand, in HLA-G the $\kappa B$ sites bind only the p50 subunit of $\mathrm{NF}-\kappa \mathrm{B}$, meaning the promoter is not activated by NF- $\mathrm{\kappa B}$, and part of the ISRE is deleted removing the sensitivity to IRF1 and meaning HLA-G is not induced by IFN- $\gamma$ either. ${ }^{64}$ HLA-G appears to be completely non-responsive to NLRC5, although it is induced by the DNA demethylating agent 5-aza-2'-deoxycytidine ${ }^{69}$ Distinctly, the HLA-G promoter region comprises heat shock response element (HSE), hypoxia stimulation response element (HSE), Ras response element and progesterone response element, showing that expression of HLA-G is regulated by several factors. This may give an advantage to HLA-G over other HLA-I antigens allowing better induction under stressful conditions.

Taken together this provides tremendous potential for transcriptional downregulation of classical HLA gene expression in cancer, perhaps with upregulation of non 
classical HLA. In line with these observations, low classical HLA expression in neuroblastoma was found to be associated with low transcriptional availability of NF-kB. ${ }^{70}$

\section{Post-transcriptional silencing}

Expression of HLA-I antigens is also controlled at the RNA level. Some HLA alleles have variable sites in the three prime untranslated region $\left(3^{\prime}\right.$ UTR $)$ that are recognized by RNA binding proteins and miRNAs. Particularly, nonclassical HLA-I molecules have been shown to be strongly regulated by miRNAs. The high tissue tropism of HLA-G is not only related to its unique promoter region but also its distinctive $3^{\prime} \mathrm{UTR}{ }^{71}$ For example, inverse correlation between miR-628-5 $\mathrm{p}$ and HLA-G expression has been identified in renal cell carcinoma. ${ }^{72}$ Upregulation of some miRNAs has also been reported to affect classical HLA-I expression by silencing HLA or components of APM. For example, Mari et al reported that overexpression of miR148a in esophageal carcinoma cell lines reduced expression of HLA-ABC. They also showed miR-125 decreased the level TAP2 which affected HLA expression. ${ }^{73}$ Similarly, Lazaridou et al found that miR200a-5p can target TAP1 and its overexpression in melanoma patients resulting in impaired HLA-I expression. ${ }^{74}$ In colorectal cancer cell lines overexpression of miR-27a repressed HLA-I expression by targeting calreticulin. ${ }^{75}$ The effects of miRNAs on cancer immune escape have been documented in more detail in a recent review by Yi $e a_{l}{ }^{76}$

Another post-transcriptional mechanism that regulates HLA-I expression is mediated by RNA binding proteins. According to a study by Huang et al, aberrant expression of the RNA binding protein MEX3B in colorectal cancer patients downregulated HLA-A expression and led to resistance to immunotherapy. In a similar vein, The Cancer Genome Atlas (TCGA) melanoma database analysis indicates that anti-PD1 resistant melanoma patient tumor samples have significantly higher MEX3B expression levels compared with the checkpoint inhibitor responsive tumors. ${ }^{77}$ Interestingly, MEX3C, which is another member of the MEX3 family, has been reported to downregulate only HLA-A by binding its 3'UTR, without affecting other class I HLAs. ${ }^{78}$ Another RNA binding protein, the heterogeneous nuclear ribonucleoprotein R (HNRNPR) has been also shown to positively regulate classical and non-classical HLA-I expression by binding to their $3^{\prime}$ UTR and stabilizing them. ${ }^{79}$

\section{Microenvironmental regulation of HLA expression}

There is a complex interplay between cancer cells and the tumor microenvironment (TME) which generally favors tumor progression by suppressing immune function. ${ }^{80}$ The best characterized microenvironmental conditions are hypoxia, acidosis, glucose deprivation and the presence of excess immunosuppressive cytokines. These conditions not only limit the activity of effector $\mathrm{T}$ cells but also dysregulate antigen presentation and HLA-I expression. ${ }^{81}$ For example, interleukin 10 (IL-10) and transforming growth factor- $\beta$ (TGF- $\beta$ ) are two major immunosuppressive cytokines that are frequently overexpressed in the TME. ${ }^{82}$ IL-10 inhibits activity of Th1 cells, NK cells, macrophages and proinflammatory cytokines, such as IFN- $\gamma$. It has also been shown that IL-10 downregulates HLA class-I molecules, suppresses antigen presentation and induces HLA-G expression in cancer cells. ${ }^{83-85}$ Similar findings have been reported for TGF- $\beta .^{86-88} \mathrm{~A}$ recent study showed that TGF- $\beta$ inhibits MHC-I expression by downregulating $\beta 2 \mathrm{M}$ via a Smad-dependent pathway. ${ }^{89}$ Lee et al examined PD1 inhibitor-resistant melanoma biopsies and found that HLA-I downregulation was associated with TGF- $\beta$ activity in $31 \%$ of progressive tumors. ${ }^{90}$

Uncontrolled tumor growth leads to insufficient blood perfusion which causes formation of hypoxic, glucose deprived and acidic conditions in the TME. Each of these conditions can induce ER stress and lead to accumulation of unfolded or misfolded proteins in the ER, which consequently, causes a significant disruption of antigen processing and presentation machinery. ${ }^{91}$ Once cells experience hypoxia, hypoxia-inducible factor 1-alpha accumulates in the cytoplasm, subsequently translocates to the nucleus and induces transcription of several genes. ${ }^{92}$ Recently, Marijt et al showed that hypoxic and glucose-deprived conditions disrupt IFN $\gamma /$ STAT1 signaling by metabolic stress-induced activation of PI3K-Akt pathway. ${ }^{93}$ An earlier study reported that transcription of HLA-I heavy chains, TAPs and LMPs were downregulated in vitro and in vivo under hypoxia, while upregulation was observed when cells were later incubated with high oxygen levels. ${ }^{94}$ Intriguingly, non-classical HLA-E and HLA-G have been shown to be overexpressed on cancer cells due to hypoxia and glucose deprivation, providing another immune escape mechanism. ${ }^{94-96}$ Cancer cells tend to produce energy by glycolysis rather than oxidative phosphorylation even in the presence of oxygen (Warburg effect). This phenomenon results in accumulation of excessive concentrations of lactate as byproduct which decreases the $\mathrm{pH}$ in the TME. Low $\mathrm{pH}$ has been shown to abrogate IFN- $\gamma$ secretion from NK cells and $\mathrm{T}$ cells which hinders IFN-mediated HLA-I expression. ${ }^{97} 98 \mathrm{~A}$ similar scenario has been recently described for the physiological regulation of $\mathrm{T}$ cells in the lymph nodes, providing further insight into the critical role of $\mathrm{pH}$ on immune function. ${ }^{99}$ Neutralization of tumor extracellular $\mathrm{pH}$ remains as an intriguing approach to improve the outcome of cancer immunotherapy. ${ }^{100} 101$

Type-I IFNs (IFN- and IFN- $\beta$ ) activate an antiviral state in cells by upregulating HLA-I expression to promote antigen presentation, ${ }^{102} 103$ while simultaneously stimulating immune cells and mediating antiproliferative effects. ${ }^{104}$ One of the major cancer-specific type-I IFN signaling defects is TME stress-mediated down-regulation of interferon alpha and beta receptor 1 (IFNAR1), ${ }^{105}$ leading to disruption of antigen presentation both in cancer and antigen presenting cells and inhibiting IFNinduced apoptosis in cancer cells. Moreover, down regulation of IFNAR1 was reported to reduce intratumoral T cell 
viability and activity due to disruption of the STAT3/granzyme B pathway. ${ }^{106}{ }^{107} \mathrm{~A}$ recent study by Cho et al showed that IFNAR1 deficient cancer-associated fibroblasts play a significant role in stromagenesis and contribute to rapid tumor growth. ${ }^{108}$ Conversely, when IFNAR1 is intact, conventional therapies can induce IFN- $\beta$ and lead to induction of HLA-I expression. ${ }^{109} 110$ For example, Wan et al observed HLA-I upregulation in breast cancer cell lines following topotecan treatment due to elevated levels of IFN- $\beta$ signaling. ${ }^{111}$ Similarly radiation induced IFN- $\beta$ led to increased HLA-I levels and subsequently sensitized cancer cells to anti-PD1 therapy, an effect that was reversed by blockade of IFNAR $1 .{ }^{112}$ IFN- $\beta$ monotherapy was also reported to increase HLA-I expression in melanoma cell lines and upregulate presentation of tumor associated antigens, such as Melan-A/Mart-1, gp100, MAGE-A1, although this effect was not observed when cells were treated with IFN- $\gamma$ or IFN-. ${ }^{113}$

\section{Oncogene-mediated regulation of HLA expression}

Oncogene activation can also modulate surface HLA expression (figure 1). For example, abnormal activity of the MAPK pathway in tumor cells has been reported to down-regulate antigen processing and presentation. ${ }^{114}$ The MAPK pathway consists of the Ras/Raf/MEK/ERK signaling cascade and can be activated by growth factor receptors, such as HER2 and EGFR, which are known to be selectively overexpressed in many tumor types. Activation of MAPK signaling, either by overexpression of growth factor receptors, or by gain-of-function mutations in signaling proteins can lead to loss of HLA. ${ }^{115-118}$ Similarly HLA downregulation and APM deficiencies were found to be more frequent in K-Ras mutated NSCLC and colorectal cancer, ${ }^{119} 120$ although application of MAPK or EGFR inhibitors could recover cell surface HLA expression and restore TAP1, TAP2, and $\beta 2 \mathrm{M} .{ }^{120}{ }^{121}$ Ras/MAPK activity has been correlated with reduced level of tumorinfiltrating lymphocytes (TILs) together with low HLA expression in triple negative breast cancer. Intriguingly, simultaneous inhibition of programmed death-ligand 1 (PD-L1) and MEK enhanced antitumor immune response in breast cancer-bearing mice. ${ }^{122}$ Similarly the oncogenic BRAF V600 mutation is very frequent in advanced melanoma and recent studies have shown that BRAF inhibitors can relieve immune suppression in TME by upregulating HLA expression. ${ }^{123}{ }^{124}$ Bradley et al reported that in melanoma cells BRAF V600E mutation drives internalization of surface HLA molecules and subsequent degradation by endocytic compartments. This process related to phosphorylation of highly conserved Serine-335 site within the HLA-I cytoplasmic tail. ${ }^{125}$ Additionally, immunosuppressive HLA-G upregulation was detected in BRAF V600E mutated papillary thyroid carcinoma. ${ }^{126}$

Along with the MAPK pathway, the PI3K-Akt pathway is also activated by receptor tyrosine kinases and frequently dysregulated in cancer cells. ${ }^{127}$ Recent studies have shown that increased signaling of the PI3K-Akt pathway down regulates class I HLA expression due to a complex interplay between PI3K and STAT1. ${ }^{128} 129$ As discussed above, hypoxic and glucose-deprived conditions have been shown to induce PI3K-Akt activation, which may contribute to the loss of HLA in cancers. ${ }^{93}$ Pharmacological inhibition of PI3K restored IFN $\gamma /$ STAT1 signaling transduction and surface class I HLA expression in vitro. ${ }^{93} 129$ A study by Sivaram et al investigating the impact of mutational activation of PI3K in an orthotopic pancreas mouse model showed that inhibition of the PI3K pathway increases MHC-I expression, leading to tumor regression due to $\mathrm{T}$ cell infiltration. ${ }^{130}$ Importantly TCGA database analysis also reveals a negative correlation between activated PI3K-Akt and HLA-I-mediated regression in head and neck cancer, lung squamous carcinoma, and pancreatic adenocarcinoma. ${ }^{129} 130$

\section{Post-translational silencing}

Recently, Yamamoto et al described another mechanism that cancer cells exploit to downregulate HLA-I expression. Even in tumors with intact HLA and APM genes, surface MHC levels could be significantly reduced by selectively targeting HLA-I molecules for lysosomal degradation via an autophagy dependent mechanism. Inhibition of autophagy using the anti-malaria drug chloroquine restored the cell surface MHC expression, improved antitumor $\mathrm{T}$ cell response in animals and synergized with immune checkpoint therapy. ${ }^{131}$

\section{Cancer stem cells and HLA}

Cancer stem cells (CSC) are thought to represent a minor component of many tumors, described to have crucial roles in formation, sustenance, metastasis and recurrence of the disease. ${ }^{132}$ A growing number of studies suggests that complete recovery can only be possible when CSC are eradicated. ${ }^{133}$ While the interaction between CSC and immune function remains to be explored, it has been suggested that they can evade immune surveillance by down regulating HLA-I expression. ${ }^{134}$ Morrison et al developed a CSC-enriched murine lung tumor sphere model and compared it with non-CSCs counterparts in terms of class I MHC expression level and susceptibility to antitumor immune response. They found that CSCenriched tumors had lower MHC expression and were more aggressive in mice. ${ }^{135}$ In clinical settings, loss of class I HLA in CSC has been reported in surgical samples of glioblastoma, melanoma and colorectal cancers. ${ }^{136-138}$ Yang et al showed that HLA-I down regulation in glioma stem cells was associated with aberrant Wnt/ $\beta$-catenin activity, which is a physiological regulator of pluripotency and self-renewal of stem cells. Treatment with HDAC inhibitors restored HLA-I expression partly by inactivating Wnt/ $\beta$-catenin. ${ }^{139}$ Importantly, while ex vivo experiments have shown that CSC and also cancer cells can be susceptible to NK cell-mediated killing due to low HLA-I expression, ${ }^{137} 140141$ these cells still survive and promote tumorigenesis in vivo, indicating the presence of another immune escape mechanism for CSC. In the next section, 
we discuss this pathological phenomenon of resistance of tumor cells to NK cell-mediated killing.

\section{Upregulation of non-classical HLA-I molecules $E, F$ and $G$}

Tumorigenesis and fetal development show several similarities in their life cycles. ${ }^{142}$ Even though tumors are a complex process of unplanned cellular and molecular dysregulations, while pregnancy is a programmed physiological process, they both develop strategies to avoid immune detection while surviving in an oxygen-deficient microenvironment. Non-classical HLA-E, HLA-F and HLA-G can suppress immune cells by interacting with inhibitory receptors. Physiologically they are upregulated in placenta or trophoblast and help to protect the embryo against attack by the maternal immune system. Growing evidence indicates that cancer cells exploit this same phenomenon-to evade immune detection they not only down regulate classical HLA-I antigens, they also upregulate non-classical ones similar to fetal tissues.

\section{Expression of HLA-E}

HLA-E is a non-classical HLA class I heavy chain paralogue that exists on the cell surface as a trimer with $\beta 2 \mathrm{M}$ and a bound peptide for presentation. Under normal conditions HLA-E selectively binds and presents the peptides produced following post-translational cleavage of the conserved leader sequences associated with the other class I HLA molecules (including HLA-G) and its main function appears to be in binding the inhibitory receptor NKG2A/CD94 on NK and T cells, thereby allowing NK cells indirectly to monitor expression of the other class I HLA molecules and prevent NK attack. ${ }^{8}$ There are increasing data showing that tumors can exploit expression of HLA-E as a means to avoid recognition by NK cells, even when classical class I HLA is not present. Aberrant HLA-E expression has been detected in colorectal cancer $(65 \%)$, gastric cancer $(45 \%)$, ovarian $(89 \%)$, breast $(50 \%)$ and associated with poor prognosis. ${ }^{143-146}$ In a study evaluating an anti-NKG2A antibody (monalizumab) as a novel checkpoint inhibitor, André $e t$ al observed HLA-E expression in many different tumor types, sometimes at higher levels than PD-L1. Disruption of its binding to NKG2A with monalizumab boosted T cell and NK cell activity in vitro and in vivo. ${ }^{92}$ Intriguingly, it has been also suggested that HLA-E can present pathogen-derived or tumor-derived peptides to unconventional $\mathrm{T}$ cells ${ }^{147148}$ and at least one report has shown high HLA-E expression in colorectal cancer patients associated with favorable prognosis. ${ }^{149}$

\section{Expression of HLA-F}

HLA-F is the least investigated and characterized nonclassical HLA-I antigen. Physiologically it is known to be expressed on activated B cells, T cells and placental tissues. ${ }^{150-152}$ However, recent studies have shown that it can be upregulated in cases of infection, autoimmune disorders and cancer. ${ }^{153}$ HLA-F is expressed in two forms, as an empty open conformer which lacks $\beta 2 \mathrm{M}$ association or as a complex with $\beta 2 \mathrm{M}$ and peptide, similar to classical HLA-I molecules. However, it is still uncertain what mediate the transformation between these two forms. While open conformers can be recognized by activating or inhibitory KIRs, the peptide- $\beta 2 \mathrm{M}$ complex form has been suggested to bind inhibitory receptors ILT2 and ILT $4 .{ }^{154}$ Indeed, while overexpression of HLA-F has been reported for various cancer types such as breast cancer $(40 \%)$, NSCLC (24\%), nasopharyngeal cancer (18\%), esophageal squamous carcinoma (21\%), and gastric cancer $(43 \%)$ by immunohistochemistry (IHC) analysis, the impact on disease prognosis remains controversial. ${ }^{155-160}$ It is important to note that analysis of HLA-F mRNA levels may give misleading information as HLA-F is partially retained intracellularly.

\section{Expression of HLA-G}

HLA-G expression in tumors has been described as another immune escape mechanism due to its negative regulation of both adaptive and innate immunity. ${ }^{161}$ HLA-G binds to several receptors, such as KIR2DL4 (expressed by NK cells), ILT4 (expressed by APC) and ILT2 (expressed by all immune cell subsets) ${ }^{162}$ and has been implicated in multiple stages of immunoediting. ${ }^{163}$ Notably, HLA-G expression is induced under hypoxia, which is often present in advanced cancer, ${ }^{95} 164$ and overexpression has been reported for several cancer types, including esophageal squamous cell carcinoma $(90 \%)$, colorectal cancer $(70 \%)$, hepatocellular carcinoma $(65 \%)$, glioma $(70 \%)$, breast cancer $(66 \%)$, gastric cancer $(75 \%)$, melanoma $(36 \%)$, ovarian cancer $(55 \%)$, and lung cancer $(75 \%) .{ }^{165-172}$ Besides, HLA-G mRNA expression data provided from a TCGA database cohort confirmed that $H L A-G$ gene is expressed in several tumors. A correlation between high proinflammatory transcripts and high HLA-G mRNA expression was used to infer that HLA-G may be important in controlling immune recognition of proinflammatory events during tumor progression. ${ }^{173}$ However, HLA-G mRNA data should be interpreted cautiously as post-transcriptional regulation, particularly by miRNAs, ${ }^{174}{ }^{175}$ is thought to be central to regulating protein expression. On the other hand, a sHLA-G protein form has been detected in plasma, serum and malignant ascites of cancer patients and correlated with poor prognosis. ${ }^{176-179}$ Taken together, HLA-G has been described as a new checkpoint inhibitor and molecular target for immunotherapy. ${ }^{180}{ }^{181}$ It has already been proposed that renal carcinoma patients may benefit from anti-HLA-G/ ILT2 and anti-HLA-G/ILT4 therapy. ${ }^{182} 183$

\section{MHC-I STATUS OF PRECLINICAL ANIMAL MODELS}

Syngeneic animal cancer models are a staple component of pre-clinical development for cancer immunotherapies, however, the MHC-I status of the animal tumor cells and the degree to which this may reflect human patients is rarely discussed. Among the most popular preclinical models, B16 murine melanoma cells normally have low 
levels of basal MHC-I expression both in vitro and in vivo, but this is substantially upregulated by exposure to interferons, reversing their intrinsic insensitivity to cytotoxic T cells. ${ }^{184} 185$ Accordingly, it may not be surprizing to observe favorable efficacy in B16 models while exploring the use of immune adjuvants that trigger interferon pathways such as STING agonists. ${ }^{186-192}$ Although both MHC-I upregulation and CD8 + priming may be contributing to the therapeutic outcomes observed in these studies, the former is seldom discussed.

For melanoma patients who have a reversible IFN $\gamma$-reversible MHC (HLA) phenotype, B16 tumors might be an appropriate pre-clinical model. It follows that, ideally, patient populations of the appropriate HLA status would be recruited for clinical studies supported by relevant preclinical models. Other syngeneic models frequently used in immunotherapy studies, including CT26, RENCA, EMT6, 4T1, MC38 and EL-4, have much higher levels of basal MHC-I function relative to B16 ${ }^{193} 194$ although whether this makes them more or less relevant to the diverse population of patients is currently unclear.

When it comes to animal models of human cancer that have more durable and defined levels of dysfunction in antigen presentation, the choices are rather slim. B $78 \mathrm{H} 1$, a subclone of $\mathrm{B} 16$ is particularly interesting because its low basal MHC-I expression is not restored by gamma interferon. Furthermore, it also expresses Qa-2 the murine homologue of HLA-G. ${ }^{195}$ This could make it a very suitable model for some patients.

Overall interest in murine homologues of non-classical HLA-E and-G (known as Qa-1 and Qa-2, respectively) ${ }^{196-198}$ is extremely limited, despite their likely importance in the human setting. They are seldom considered when interpreting immunotherapy data in the most frequently used translational models.

In summary, it is not obvious that the antigen presentation status of commonly used animal models reflects the phenotypes found in clinical disease. We believe it is likely that increased focus on aligning antigen presentation competencies in laboratory models and patients could improve the effective translation of new immunotherapies.

\section{EFFECTS ON CLINICAL PRACTICE}

Insights into the critical role of the immune system in prevention of tumorigenesis paved the way for development of several immunotherapeutics, such as checkpoint inhibitors, cancer vaccines and adoptive $T$ cell therapy. During the past decade use of checkpoint inhibitors was approved by FDA for the treatment of metastatic melanoma and undoubtedly brought new hope to many. However, the number of patients who benefit from the treatment has remained low, according to a recent study the number is only about $12 \%{ }^{199}$ Various factors can influence the responsiveness to immunotherapy, and it is important to identify these factors to guide efficient development of new drugs capable of activating the immune system against the cancer.

\section{HLA-I diversity as a predictive biomarker for immunotherapy}

Tumor mutation burden (TMB) and inflammation status of the TME have been proposed as potential predictive biomarkers of response to ICIs. ${ }^{200}{ }^{201}$ However, high TMB has been detected both in ICI responsive and nonresponsive patients, indicating an important impact of other parameters. ${ }^{202}{ }^{203}$ Notably, recognition of the HLA peptide complex by the TCR is essential for T cell mediated cell killing. Illustrating clinical importance, HLA-I heterozygosity has been correlated with improved overall survival of patients who received checkpoint inhibitors, while HLA-I homozygosity in at least one locus reduced survival significantly. ${ }^{204}$ Moreover, an association between high mutational burden and better survival was found to be further increased in patients with all loci HLA-I heterozygosity, indicating the importance of diverse HLA-I expression on outcome of checkpoint blockade therapy. ${ }^{204}$ The TRACERx study showed that cancer cells with LOH at HLA loci had higher mutational burdens, however, loss of HLA-I meant that these neoantigens could not be presented to $T$ cells which could contribute toward resistance to ICIs. Other groups also observed similar correlations between high TMB and HLA-LOH, and combinational analysis of TMB and HLA-I heterozygosity has been proposed as a possible predictive biomarker for responsiveness to ICI therapy. ${ }^{205} 206$

\section{Prognostic effect of HLA-I status}

Although the vast majority of studies so far have shown that loss of HLA-I is correlated with poor overall survival, some opposite results have also been published even for the same types of cancer. For example, Hiraoka et alstated in their retrospective study that high HLA levels shorten the overall survival of patients with pancreatic ductal adenocarcinoma (PDAC), but in an earlier study, Imai $e t$ al demonstrated that HLA loss was associated with poor survival in PDAC patients. ${ }^{207} 208$ A similar discrepancy has been reported for gastric patients, ${ }^{209} 210$ but in these two studies the HLA-I levels were investigated in different stages of the disease. While in advanced stages of gastric cancer (MSI-high) HLA-I expression was not a prognostic factor, in earlier stages of gastric cancer (MSS and MSIlow) loss of HLA-I caused a worse survival rate. Reports for colorectal cancer and esophageal cancer are other examples of controversial observations in terms of HLA status and disease prognosis. ${ }^{211} 212$ The reason for high HLA expression to be associated with poor prognosis was mostly considered to relate to NK-cell inhibition. However, taken together, the most predominant observations are that loss of HLA-I combined with high PD-L1 expression levels gives poor prognosis and that there is a positive correlation between classical HLA-I expression and the density of TILs in the TME. Clinical implications of HLA-I status are summarized in table 1. 


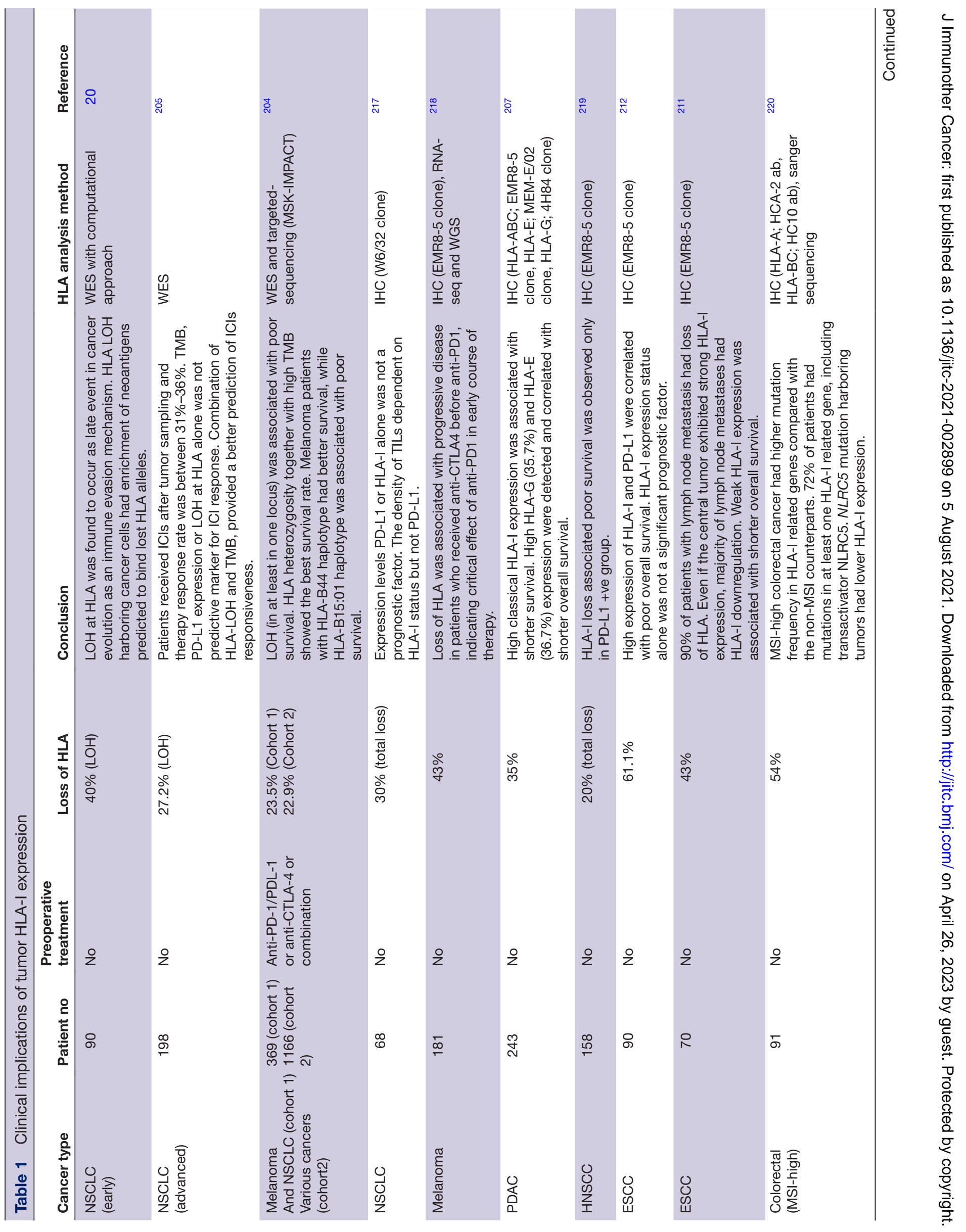




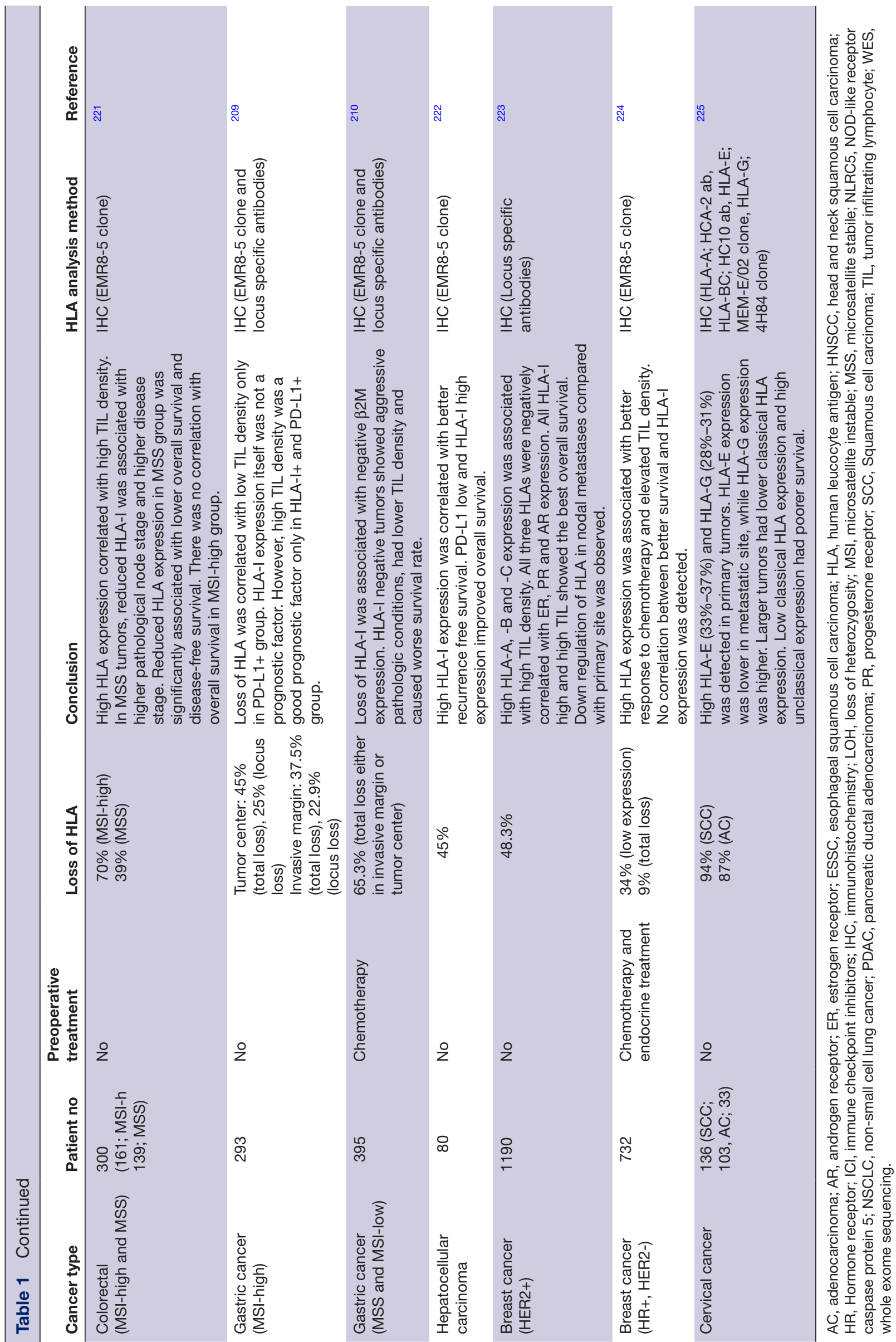




\section{HLA analysis methods in clinical studies}

Standardized protocols for detection and analysis of functional HLA are urgently needed. The most commonly used HLA analysis technique is IHC, and this gives no real indication of functionality. ${ }^{213}$ IHC tests also face several practical issues, such as preparation of tissue sections, selection of appropriate antibodies and determination of cut-off value for assessing positivity. Cut-off values are usually set according to HLA-I expression levels in tumor stroma or adjacent tissues, however, there is no established guideline for scoring and interpreting the results. Moreover, when non-classical HLA-I molecules are taken into account it becomes even more complicated. The majority of commercially available antibodies against non-classical HLA-I antigens are reported to show low specificity, meaning that HLA-E, HLA-F or HLA-G expression may be inaccurately assessed. ${ }^{214}$ On the other hand, whole genome sequencing and whole exome sequencing are next generation sequencing approaches which have been used to detect HLA-I mutations. Targeted sequencing, such as MSK-IMPACT panel is also widely used to capture HLA-A, -B and -C. ${ }^{215}$ One of the greatest advantages of these techniques over IHC is the ability to detect allelespecific losses. As mentioned above, particularly during selective pressure, HLA alleles capable of presenting neoantigens may be lost, and a change that is not detectable by IHC. The great majority of the studies, as shown in table 1, were conducted with IHC analysis, which may in fact be one of the main reasons restricting us from seeing the true picture of the consequences of HLA loss. Importantly, IHC does not give any insight into whether loss of HLA is based on irreversible or reversible defects. This simple information would provide a platform for more rational individualized treatment design.

\section{CONCLUSION}

Major advances in immunotherapy in recent years have brought new perspective to cancer treatment. However, it is now clear that the number of patients responding to immunotherapy is relatively limited. Moreover, acquired resistance in patients who initially responded to treatment reveals an important obstacle that needs to be solved. Although the resistance mechanisms developed by cancer cells against immunotherapy have not been fully characterized, cell surface HLA-I expression and antigen processing, which are indispensable for normal immune function, undoubtedly play an important role. Antigen processing and presentation is a mechanism which needs harmonious function of several different cellular compartments, providing an array of possibilities for cancer cells to disrupt the system.

Genetic mutations in HLA-I expression or in APM compartments are classified as irreversible defects. In these cases, either HLA function must be restored using gene therapy or gene editing techniques, or treatment approaches should focus on classical chemoradiotherapy or the use of HLA-independent immunotherapy, such as
CAR-T cells or bispecific T-cell engagers. Each of these approaches is showing rapid progress currently, and careful stratification of patients for HLA function should enable more precise scrutiny of their mechanisms of action and further accelerate clinical progress.

The more common mechanisms of HLA-I losses are reversible defects, involving transcriptional, posttranscriptional or epigenetic changes, where the HLA-I level may sometimes be restored by pharmacological intervention. Particularly, the application of type-I or type-II interferons often results in a significant increase in HLA-I levels in many cells with such reversible defects. Likewise, in recent years, preclinical epigenetic therapy approaches, such as HDAC inhibition, have also been shown to increase cell surface HLA-I levels and to exhibit synergistic effects with immunotherapy.

The other side of the medallion reflects the nonclassical HLA-I antigens, whose impact on tumorigenesis remains controversial. The number of reports indicating a role of non-classical HLA-I molecules on cancer development and immunoediting is increasing rapidly. Some studies even suggest that HLA-E or HLA-G are expressed at higher levels than PD-L1 and therefore may play a substantial regulatory role as immune checkpoints. ${ }^{181} 216$ In future clinical studies, HLA-E and HLA-G levels should be routinely analyzed to generate more extensive data to underpin rational treatment strategies. Undoubtedly, the critical point here is that a standardized analysis protocol has not yet been established for detection of classical and non-classical HLA-I antigens. There is also an urgent need for development of HLA-E and HLA-G specific monoclonal antibodies to be used routinely in clinical settings.

Immunotherapy, like most cancer drugs, can cause serious undesired side effects. Checkpoint inhibitor antibodies have become the most applied type of immunotherapy in the clinic and also the most widely investigated immunotherapy approach in preclinical studies. Most studies focus on how to increase the efficiency of checkpoint inhibitors or to determine which other treatments can create synergistic effects to enhance their benefit. However, still there is a need for predictive biomarkers for selection of patients who will receive the therapy. Although HLA-I expression profile alone does not seem to be a sufficient parameter for successful ICI response in most of the clinical studies, it has a predictive value when tumor PD-L1 levels and TMB are considered together. However, most of the clinical observations are made on the basis of small biopsy materials from single tumor sites, despite the heterogeneous nature of cancer. In addition, the fact that a functional HLA-I analysis method has not yet been established probably underpins the poor understanding of the real effect of classical HLA-I loss or nonclassical HLA-I upregulation on patients' response to immunotherapy.

Contributors All of the authors contributed to the production of ideas supporting this manuscript and each of them was also involved in helping to write the manuscript. 
Funding This study was funded by Cancer Research UK (C552/A29106).

Competing interests None declared.

Patient consent for publication Not required.

Provenance and peer review Not commissioned; externally peer reviewed.

Open access This is an open access article distributed in accordance with the Creative Commons Attribution Non Commercial (CC BY-NC 4.0) license, which permits others to distribute, remix, adapt, build upon this work non-commercially, and license their derivative works on different terms, provided the original work is properly cited, appropriate credit is given, any changes made indicated, and the use is non-commercial. See http://creativecommons.org/licenses/by-nc/4.0/.

\section{ORCID iD}

Len Seymour http://orcid.org/0000-0003-3825-0841

\section{REFERENCES}

1 Bukur J, Jasinski S, Seliger B. The role of classical and nonclassical HLA class I antigens in human tumors. Semin Cancer Biol 2012:22:350-8.

2 Cornel AM, Mimpen IL, Nierkens S. Mhc class I downregulation in cancer: underlying mechanisms and potential targets for cancer immunotherapy. Cancers 2020;12:1760.

3 Anderson P, Aptsiauri N, Ruiz-Cabello F, et al. Hla class I loss in colorectal cancer: implications for immune escape and immunotherapy. Cell Mol Immunol 2021;18:556-65.

4 Garrido F, Aptsiauri N. Cancer immune escape: MHC expression in primary tumours versus metastases. Immunology 2019;158:255-66.

5 thor Straten P, Garrido F. Targetless T cells in cancer immunotherapy. J Immunother Cancer 2016;4:23.

6 Parker KC, DiBrino M, Hull L. The beta 2-microglobulin dissociation rate is an accurate measure of the stability of MHC class I heterotrimers and depends on which peptide is bound. The Journal of Immunology 1992;149:1896-904.

7 Boudreau JE, Hsu KC. Natural killer cell education in human health and disease. Curr Opin Immunol 2018;50:102-11.

8 Kaiser BK, Pizarro JC, Kerns J, et al. Structural basis for NKG2A/CD94 recognition of HLA-E. Proc Natl Acad Sci U S A 2008;105:6696-701.

9 Braud VM, Allan DSJ, Wilson D, et al. TAP- and tapasin-dependent HLA-E surface expression correlates with the binding of an $\mathrm{MHC}$ class I leader peptide. Curr Biol 1998;8:1-10.

10 Kovats S, Main E, Librach C, et al. A class I antigen, HLA-G, expressed in human trophoblasts. Science 1990;248:220-3.

11 Shobu T, Sageshima N, Tokui H, et al. The surface expression of HLA-F on decidual trophoblasts increases from mid to term gestation. J Reprod Immunol 2006;72:18-32.

12 Lee N, Geraghty DE. Hla-F surface expression on B cell and monocyte cell lines is partially independent from tapasin and completely independent from TAP. J Immunol 2003;171:5264-71.

13 HoWangYin K-Y, Loustau M, Wu J, et al. Multimeric structures of HLA-G isoforms function through differential binding to LILRB receptors. Cellular and Molecular Life Sciences 2012;69:4041-9.

14 Amiot L, Ferrone S, Grosse-Wilde H, et al. Biology of HLA-G in cancer: a candidate molecule for therapeutic intervention? Cell Mol Life Sci 2011;68:417-31.

15 Hofmeister V, Weiss EH. Hla-G modulates immune responses by diverse receptor interactions. Semin Cancer Biol 2003;13:317-23.

16 Dunn GP, Bruce AT, Ikeda H, et al. Cancer immunoediting: from immunosurveillance to tumor escape. Nat Immunol 2002;3:991-8.

17 de Bruin EC, McGranahan N, Mitter R, et al. Spatial and temporal diversity in genomic instability processes defines lung cancer evolution. Science 2014;346:251-6.

18 Garrido F. MHC/HLA class I loss in cancer cells. Adv Exp Med Biol 2019;1151:15-78.

19 Huang Jian'An, Hu J, Fu X. The prevalence of HLA LOH across 10 cancer types in Chinese patients. J Clin Oncol 2020;38:3124.

20 McGranahan N, Rosenthal R, Hiley CT, et al. Allele-Specific HLA loss and immune escape in lung cancer evolution. Cell 2017;171:1259-71. e1211.

21 Shukla SA, Rooney MS, Rajasagi M, et al. Comprehensive analysis of cancer-associated somatic mutations in class I HLA genes. Nat Biotechnol 2015;33:1152-8.

22 Kageshita T, Kawakami Y, Ono T. Clinical significance of MART-1 and HLA-A2 expression and CD8+ T cell infiltration in melanocytic lesions in HLA-A2 phenotype patients. J Dermatol Sci 2001;25:36-44.
23 Rosenberg SA, Tran E, Robbins PF. T-Cell transfer therapy targeting mutant KRAS. N Engl J Med 2017;376:e11.

24 Paschen A, Méndez RM, Jimenez P, et al. Complete loss of HLA class I antigen expression on melanoma cells: a result of successive mutational events. Int J Cancer 2003;103:759-67.

25 del Campo AB, Kyte JA, Carretero J, et al. Immune escape of cancer cells with beta2-microglobulin loss over the course of metastatic melanoma. Int J Cancer 2014;134:102-13.

26 Castro A, Ozturk K, Pyke RM, et al. Elevated neoantigen levels in tumors with somatic mutations in the HLA-A, HLA-B, HLA-C and B2M genes. BMC Med Genomics 2019;12:107.

27 Sade-Feldman M, Jiao YJ, Chen JH, et al. Resistance to checkpoint blockade therapy through inactivation of antigen presentation. Nat Commun 2017;8:1136.

28 Barrow P, Richman SD, Wallace AJ, et al. Confirmation that somatic mutations of beta-2 microglobulin correlate with a lack of recurrence in a subset of stage II mismatch repair deficient colorectal cancers from the QUASAR trial. Histopathology 2019;75:236-46.

29 Maleno I, Aptsiauri N, Cabrera T, et al. Frequent loss of heterozygosity in the $\beta 2$-microglobulin region of chromosome 15 in primary human tumors. Immunogenetics 2011;63:65-71.

30 Zaretsky JM, Garcia-Diaz A, Shin DS, et al. Mutations associated with acquired resistance to PD-1 blockade in melanoma. $N$ Engl $J$ Med 2016;375:819-29.

31 Gettinger S, Choi J, Hastings K, et al. Impaired HLA class antigen processing and presentation as a mechanism of acquired resistance to immune checkpoint inhibitors in lung cancer. Cancer Discov 2017;7:1420-35.

32 Le DT, Durham JN, Smith KN, et al. Mismatch repair deficiency predicts response of solid tumors to PD-1 blockade. Science 2017;357:409-13.

33 Sade-Feldman M, Yizhak K, Bjorgaard SL, et al. Defining T cell states associated with response to checkpoint immunotherapy in melanoma. Cell 2019;176:404

34 Detjen KM, Farwig K, Welzel M, et al. Interferon gamma inhibits growth of human pancreatic carcinoma cells via caspase-1 dependent induction of apoptosis. Gut 2001;49:251-62.

35 Chawla-Sarkar M, Leaman DW, Borden EC. Preferential induction of apoptosis by interferon (IFN)-beta compared with IFN-alpha2 correlation with TRAIL/Apo2L induction in melanoma cell lines. Clin Cancer Res 2001;7:1821-31.

36 Abril E, Mendez RE, García A, et al. Characterization of a gastric tumor cell line defective in MHC class I inducibility by both $\alpha$ - and $\gamma$-interferon. Tissue Antigens 1996;47:391-8.

37 Meissner TB, Li A, Biswas A, et al. NIr family member NLRC5 is a transcriptional regulator of $\mathrm{MHC}$ class I genes. Proc Natl Acad Sci U S A 2010;107:13794-9.

$38 \mathrm{Hu} \mathrm{X}$, Ivashkiv LB. Cross-Regulation of signaling pathways by interferon-gamma: implications for immune responses and autoimmune diseases. Immunity 2009;31:539-50.

39 Gao J, Shi LZ, Zhao H, et al. Loss of IFN- $\gamma$ pathway genes in tumor cells as a mechanism of resistance to anti-CTLA-4 therapy. Cell 2016;167:397-404.

40 Shin DS, Zaretsky JM, Escuin-Ordinas H, et al. Primary Resistance to PD-1 Blockade Mediated by JAK1/2 Mutations. Cancer Discov 2017;7:188-201.

41 Kalbasi A, Tariveranmoshabad M, Hakimi K, et al. Uncoupling interferon signaling and antigen presentation to overcome immunotherapy resistance due to JAK1 loss in melanoma. Sci Trans/ Med 2020;12. doi:10.1126/scitransImed.abb0152. [Epub ahead of print: 14102020$]$.

42 Zhang $\mathrm{C}$, Hou D, Wei $\mathrm{H}$, et al. Lack of interferon- $\gamma$ receptor results in a microenvironment favorable for intestinal tumorigenesis. Oncotarget 2016;7:42099-109.

43 Ikeda H, Old LJ, Schreiber RD. The roles of IFN gamma in protection against tumor development and cancer immunoediting. Cytokine Growth Factor Rev 2002;13:95-109.

44 Ferrington DA, Gregerson DS. Immunoproteasomes: structure, function, and antigen presentation. Prog Mol Biol Trans/ Sci 2012;109:75-112

45 Mehta AM, Osse M, Kolkman-Uljee S, et al. Molecular backgrounds of ERAP1 downregulation in cervical carcinoma. Anal Cell Pathol 2015;2015:367837:1-5.

46 Shionoya Y, Kanaseki T, Miyamoto S, et al. Loss of tapasin in human lung and colon cancer cells and escape from tumorassociated antigen-specific CTL recognition. Oncoimmunology 2017;6:e1274476.

47 Subramaniam D, Thombre R, Dhar A, et al. Dna methyltransferases: a novel target for prevention and therapy. Front Oncol 2014;4:80. 
48 Ramsuran V, Kulkarni S, O'huigin C, et al. Epigenetic regulation of differential HLA-A allelic expression levels. Hum Mol Genet 2015;24:4268-75.

49 van den Elsen PJ. Expression regulation of major histocompatibility complex class I and class II encoding genes. Front Immunol 2011;2:48

50 Stone ML, Chiappinelli KB, Li H, et al. Epigenetic therapy activates type I interferon signaling in murine ovarian cancer to reduce immunosuppression and tumor burden. Proc Natl Acad Sci U S A 2017;114:E10981-90.

$51 \mathrm{Xi} \mathrm{S}$, Dyer KF, Kimak M, et al. Decreased STAT1 expression by promoter methylation in squamous cell carcinogenesis. J Natl Cancer Inst 2006;98:181-9.

52 Sun T, Li Y, Yang W, et al. Histone deacetylase inhibition upregulates $\mathrm{MHC}$ class I to facilitate cytotoxic T lymphocyte-mediated tumor cell killing in glioma cells. J Cancer 2019;10:5638-45.

53 Llopiz D, Ruiz M, Villanueva L, et al. Enhanced anti-tumor efficacy of checkpoint inhibitors in combination with the histone deacetylase inhibitor belinostat in a murine hepatocellular carcinoma model. Cancer Immunol Immunother 2019;68:379-93.

54 Terranova-Barberio M, Thomas S, Ali N, et al. Hdac inhibition potentiates immunotherapy in triple negative breast cancer. Oncotarget 2017;8:114156-72.

55 Knox T, Sahakian E, Banik D, et al. Selective HDAC6 inhibitors improve anti-PD-1 immune checkpoint blockade therapy by decreasing the anti-inflammatory phenotype of macrophages and down-regulation of immunosuppressive proteins in tumor cells. Sci Rep 2019:9:6136.

56 Luo N, Nixon MJ, Gonzalez-Ericsson PI, et al. Dna methyltransferase inhibition upregulates $\mathrm{MHC}$-I to potentiate cytotoxic T lymphocyte responses in breast cancer. Nat Commun 2018;9:248

57 Wrangle J, Wang W, Koch A, et al. Alterations of immune response of non-small cell lung cancer with Azacytidine. Oncotarget 2013;4:2067-79.

58 Mazzone R, Zwergel C, Mai A, et al. Epi-drugs in combination with immunotherapy: a new avenue to improve anticancer efficacy. Clin Epigenetics 2017;9:59.

59 Jespersen H, Olofsson Bagge R, Ullenhag G, et al. Phase II multicenter open label study of pembrolizumab and entinostat in adult patients with metastatic uveal melanoma (PEMDAC study). Ann Oncol 2019;30:v907.

60 Agarwala SS, Moschos SJ, Johnson ML, et al. Efficacy and safety of entinostat (ENT) and pembrolizumab (PEMBRO) in patients with melanoma progressing on or after a PD-1/L1 blocking antibody. $J$ Clin Oncol 2018;36:9530.

61 Gandhi L, Janne PA, Opyrchal M, et al. Efficacy and safety of entinostat (ENT) and pembrolizumab (PEMBRO) in patients with non-small cell lung cancer (NSCLC) previously treated with anti-PD(L)1 therapy. J Clin Oncol 2018;36:9036.

62 Buenrostro JD, Giresi PG, Zaba LC, et al. Transposition of native chromatin for fast and sensitive epigenomic profiling of open chromatin, DNA-binding proteins and nucleosome position. Nat Methods 2013;10:1213-8.

63 Jongsma MLM, Guarda G, Spaapen RM. The regulatory network behind MHC class I expression. Mol Immunol 2019;113:16-21.

64 Gobin SJ, van den Elsen PJ. Transcriptional regulation of the MHC class Ib genes HLA-E, HLA-F, and HLA-G. Hum Immunol 2000;61:1102-7.

65 Vijayan S, Sidiq T, Yousuf S, et al. Class I transactivator, NLRC5: a central player in the MHC class I pathway and cancer immune surveillance. Immunogenetics 2019;71:273-82.

66 Yoshihama S, Roszik J, Downs I, et al. NLRC5/MHC class I transactivator is a target for immune evasion in cancer. Proc Natl Acad Sci U S A 2016:113:5999-6004.

67 Romero I, Martinez M, Garrido C, et al. The tumour suppressor FHIT positively regulates MHC class I expression on cancer cells. $J$ Pathol 2012;227:367-79.

68 Pulido M, Chamorro V, Romero I, et al. Restoration of MHC-I on tumor cells by FHIT transfection promotes immune rejection and acts as an individualized immunotherapeutic vaccine. Cancers 2020;12:1563.

69 Poláková K, Bandzuchová E, Kuba D, et al. Demethylating agent 5-aza-2'-deoxycytidine activates HLA-G expression in human leukemia cell lines. Leuk Res 2009;33:518-24.

70 Forloni M, Albini S, Limongi MZ, et al. Nf-kappaB, and not MYCN, regulates $\mathrm{MHC}$ class I and endoplasmic reticulum aminopeptidases in human neuroblastoma cells. Cancer Res 2010;70:916-24.

71 Reches A, Berhani O, Mandelboim O. A unique regulation region in the $3^{\prime}$ UTR of HLA-G with a promising potential. Int $J \mathrm{Mol} \mathrm{Sci}$ 2020;21:900.
72 Jasinski-Bergner S, Reches A, Stoehr C, et al. Identification of novel microRNAs regulating HLA-G expression and investigating their clinical relevance in renal cell carcinoma. Oncotarget 2016;7:26866-78.

73 Mari L, Hoefnagel SJM, Zito D, et al. microRNA 125a regulates MHC-I expression on esophageal adenocarcinoma cells, associated with suppression of antitumor immune response and poor outcomes of patients. Gastroenterology 2018;155:784-98.

74 Lazaridou MF, Gonschorek E, Massa C, et al. Identification of miR200a-5p targeting the peptide transporter TAP1 and its association with the clinical outcome of melanoma patients. Oncoimmunology 2020:9:1774323.

75 Colangelo T, Polcaro G, Ziccardi P, et al. Proteomic screening identifies calreticulin as a miR-27a direct target repressing MHC class I cell surface exposure in colorectal cancer. Cell Death Dis 2016;7:e2120.

$76 \mathrm{Yi} \mathrm{M}, \mathrm{Xu} \mathrm{L}$, Jiao Y, et al. The role of cancer-derived microRNAs in cancer immune escape. J Hematol Oncol 2020;13:25.

77 Huang L, Malu S, McKenzie JA, et al. The RNA-binding protein MEX3B mediates resistance to cancer immunotherapy by downregulating HLA-A expression. Clin Cancer Res 2018;24:3366-76.

78 Cano F, Rapiteanu R, Sebastiaan Winkler G, et al. A non-proteolytic role for ubiquitin in deadenylation of MHC-I mRNA by the RNAbinding E3-ligase MEX-3C. Nat Commun 2015;6:8670.

79 Reches A, Nachmani D, Berhani O, et al. HNRNPR regulates the expression of classical and nonclassical MHC class I proteins. $J$ Immunol 2016:196:4967-76.

80 Quail DF, Joyce JA. Microenvironmental regulation of tumor progression and metastasis. Nat Med 2013;19:1423-37.

81 Lim AR, Rathmell WK, Rathmell JC. The tumor microenvironment as a metabolic barrier to effector T cells and immunotherapy. eLife 2020;9:e55185.

82 Jarnicki AG, Lysaght J, Todryk S, et al. Suppression of Antitumor Immunity by IL- 10 and TGF- $\beta$-Producing T Cells Infiltrating the Growing Tumor: Influence of Tumor Environment on the Induction of $\mathrm{CD}^{+}{ }^{+}$and CD8 ${ }^{+}$Regulatory T Cells. J Immunol 2006;177:896-904.

83 Mittal SK, Roche PA. Suppression of antigen presentation by IL-10. Curr Opin Immunol 2015;34:22-7.

84 Yue FY, Dummer R, Geertsen R, et al. Interleukin-10 is a growth factor for human melanoma cells and down-regulates HLA class-I, HLA class-II and ICAM-1 molecules. Int J Cancer 1997;71:630-7.

85 Urosevic M, Willers J, Mueller B, et al. Hla-G protein up-regulation in primary cutaneous lymphomas is associated with interleukin-10 expression in large cell T-cell lymphomas and indolent B-cell lymphomas. Blood 2002;99:609-17.

86 Berglund AK, Fisher MB, Cameron KA, et al. Transforming growth factor- $\beta 2$ downregulates major histocompatibility complex (MHC) I and $\mathrm{MHC}$ || surface expression on equine bone marrow-derived mesenchymal stem cells without altering other phenotypic cell surface markers. Front Vet Sci 2017:4:84

87 Chen X-H, Liu Z-C, Zhang G, et al. TGF- $\beta$ and EGF induced HLA-I downregulation is associated with epithelial-mesenchymal transition (EMT) through upregulation of snail in prostate cancer cells. Mol Immunol 2015;65:34-42.

88 Guan Z, Song B, Liu F, et al. TGF- $\beta$ induces HLA-G expression through inhibiting miR-152 in gastric cancer cells. J Biomed Sci 2015;22:107.

89 Berglund AK, Long JM, Schnabel LV. TGF- $\beta$ downregulates MHC I surface expression through a Smad3-dependent mechanism. $J$ Immunol 2020;204:140.142.

90 Lee JH, Shklovskaya E, Lim SY, et al. Transcriptional downregulation of MHC class I and melanoma de- differentiation in resistance to PD-1 inhibition. Nat Commun 2020;11:11.

91 Granados DP, Tanguay P-L, Hardy M-P, et al. Er stress affects processing of MHC class I-associated peptides. BMC Immunol 2009;10:10.

92 André $P$, Denis $C$, Soulas $C$, et al. Anti-NKG2A mAb is a checkpoint inhibitor that promotes anti-tumor immunity by Unleashing both $\mathrm{T}$ and NK cells. Cell 2018:175:1731-43. e1713.

93 Marijt KA, Sluijter M, Blijleven L, et al. Metabolic stress in cancer cells induces immune escape through a PI3K-dependent blockade of IFN $\gamma$ receptor signaling. J Immunother Cancer 2019;7:152.

94 Sethumadhavan S, Silva M, Philbrook P, et al. Hypoxia and hypoxiainducible factor (HIF) downregulate antigen-presenting MHC class I molecules limiting tumor cell recognition by T cells. PLoS One 2017;12:e0187314.

95 Mouillot G, Marcou C, Zidi I, et al. Hypoxia modulates HLA-G gene expression in tumor cells. Hum Immunol 2007;68:277-85.

96 Yaghi L, Poras I, Simoes RT, et al. Hypoxia inducible factor-1 mediates the expression of the immune checkpoint HLA-G in 
glioma cells through hypoxia response element located in exon 2. Oncotarget 2016;7:63690-707.

97 Husain Z, Huang Y, Seth P, et al. Tumor-Derived lactate modifies antitumor immune response: effect on myeloid-derived suppressor cells and NK cells. J.i. 2013;191:1486-95.

98 Calcinotto A, Filipazzi P, Grioni M, et al. Modulation of microenvironment acidity reverses anergy in human and murine tumor-infiltrating T lymphocytes. Cancer Res 2012;72:2746-56.

$99 \mathrm{Wu} \mathrm{H}$, Estrella V, Beatty M, et al. T-Cells produce acidic niches in lymph nodes to suppress their own effector functions. Nat Commun 2020;11:4113.

100 Pilon-Thomas S, Kodumudi KN, El-Kenawi AE, et al. Neutralization of tumor acidity improves antitumor responses to immunotherapy. Cancer Res 2016;76:1381-90.

101 Jin H-S, Choi D-S, Ko M, et al. Extracellular pH modulating injectable gel for enhancing immune checkpoint inhibitor therapy. $J$ Control Release 2019;315:65-75.

102 Welsh RM, Bahl K, Marshall HD, et al. Type 1 interferons and antiviral CD8 T-cell responses. PLoS Pathog 2012;8:e1002352.

103 Murata M, Nabeshima S, Kikuchi K, et al. A comparison of the antitumor effects of interferon-alpha and beta on human hepatocellular carcinoma cell lines. Cytokine 2006;33:121-8.

104 Dunn GP, Koebel CM, Schreiber RD. Interferons, immunity and cancer immunoediting. Nat Rev Immunol 2006;6:836-48.

105 Araya RE, Goldszmid RS. Ifnar1 degradation: a new mechanism for tumor immune evasion? Cancer Cell 2017;31:161-3.

106 Katlinski KV, Gui J, Katlinskaya YV, et al. Inactivation of interferon receptor promotes the establishment of immune privileged tumor microenvironment. Cancer Cell 2017;31:194-207.

107 Lu C, Klement JD, Ibrahim ML, et al. Type I interferon suppresses tumor growth through activating the STAT3-granzyme B pathway in tumor-infiltrating cytotoxic T lymphocytes. J Immunother Cancer 2019;7:157

108 Cho C, Mukherjee R, Peck AR, et al. Cancer-Associated fibroblasts downregulate type I interferon receptor to stimulate intratumoral stromagenesis. Oncogene 2020;39:6129-37.

109 Ohtsukasa S, Okabe S, Yamashita H, et al. Increased expression of CEA and MHC class I in colorectal cancer cell lines exposed to chemotherapy drugs. J Cancer Res Clin Oncol 2003;129:719-26.

110 Fonsatti E, Nicolay HJM, Sigalotti L, et al. Functional up-regulation of human leukocyte antigen class I antigens expression by 5-aza2 '-deoxycytidine in cutaneous melanoma: immunotherapeutic implications. Clin Cancer Res 2007:13:3333-8.

111 Wan S, Pestka S, Jubin RG, et al. Chemotherapeutics and radiation stimulate $\mathrm{MHC}$ class I expression through elevated interferon-beta signaling in breast cancer cells. PLoS One 2012;7:e32542.

112 Wang X, Schoenhals JE, Li A, et al. Suppression of type I IFN signaling in tumors mediates resistance to anti-PD- 1 treatment that can be overcome by radiotherapy. Cancer Res 2017;77:839-50.

113 Dunn IS, Haggerty TJ, Kono M, et al. Enhancement of human melanoma antigen expression by IFN-beta. J Immunol 2007;179:2134-42.

114 Kono M, Dunn IS, Durda PJ, et al. Role of the mitogen-activated protein kinase signaling pathway in the regulation of human melanocytic antigen expression. Mol Cancer Res 2006;4:779-92.

115 Mimura K, Ando T, Poschke I, et al. T cell recognition of HLA-A2 restricted tumor antigens is impaired by the oncogene HER2. Int $J$ Cancer 2011;128:390-401.

116 Vertuani S, Triulzi C, Roos AK, et al. Her-2/Neu mediated downregulation of $\mathrm{MHC}$ class I antigen processing prevents $\mathrm{CTL}-$ mediated tumor recognition upon DNA vaccination in HLA-A2 transgenic mice. Cancer Immunol Immunother 2009;58:653-64.

117 Brea EJ, Oh CY, Manchado E, et al. Kinase regulation of human MHC class I molecule expression on cancer cells. Cancer Immunol Res 2016;4:936-47.

118 Concha-Benavente F, Srivastava RM, Ferrone S. Egfr-Mediated tumor immunoescape: the imbalance between phosphorylated STAT1 and phosphorylated STAT3. Oncoimmunology 2013;2:e27215

$119 \mathrm{He}$ XP, Song FJ, Liu XY. The relationship between KRAS gene mutations and HLA class I antigen downregulation in the metastasis of non-small cell lung cancer. $J$ Int Med Res 2013;41:1473-83.

120 Mimura K, Shiraishi K, Mueller A, et al. The MAPK pathway is a predominant regulator of HLA-A expression in esophageal and gastric cancer. J Immunol 2013;191:6261-72.

121 Herrmann F, Lehr H-A, Drexler I, et al. HER-2/neu-mediated regulation of components of the MHC class I antigen-processing pathway. Cancer Res 2004;64:215-20.

122 Loi S, Dushyanthen S, Beavis PA, et al. Ras/Mapk activation is associated with reduced tumor-infiltrating lymphocytes in triplenegative breast cancer: therapeutic cooperation between MEK and PD-1/PD-L1 immune checkpoint inhibitors. Clin Cancer Res 2016:22:1499-509.

123 Sapkota B, Hill CE, Pollack BP. Vemurafenib enhances $\mathrm{MHC}$ induction in BRAF ${ }^{\mathrm{V} 600 \mathrm{E}}$ homozygous melanoma cells. Oncoimmunology 2013;2:e22890.

124 Liu L, Mayes PA, Eastman S, et al. The BRAF and MEK inhibitors dabrafenib and trametinib: effects on immune function and in combination with immunomodulatory antibodies targeting PD-1, PD-L1, and CTLA-4. Clin Cancer Res 2015;21:1639-51.

125 Bradley SD, Chen Z, Melendez B, et al. BRAF ${ }^{6600 E}$ Co-opts a Conserved MHC Class I Internalization Pathway to Diminish Antigen Presentation and CD8 ${ }^{+}$T-cell Recognition of Melanoma. Cancer Immunol Res 2015;3:602-9.

126 Smallridge RC, Chindris A-M, Asmann YW, et al. Rna sequencing identifies multiple fusion transcripts, differentially expressed genes, and reduced expression of immune function genes in BRAF (V600E) mutant vs BRAF wild-type papillary thyroid carcinoma. $J$ Clin Endocrinol Metab 2014;99:E338-47.

127 Thorpe LM, Yuzugullu H, Zhao JJ. Pi3K in cancer: divergent roles of isoforms, modes of activation and therapeutic targeting. Nat Rev Cancer 2015;15:7-24.

128 Nguyen H, Ramana CV, Bayes J, et al. Roles of phosphatidylinositol 3-kinase in interferon-gamma-dependent phosphorylation of STAT1 on serine 727 and activation of gene expression. $J$ Biol Chem 2001;276:33361-8.

129 Chandrasekaran S, Sasaki M, Scharer CD, et al. Phosphoinositide 3-kinase signaling can modulate MHC class I and II expression. Mol Cancer Res 2019;17:2395-409.

130 Sivaram N, McLaughlin PA, Han HV, et al. Tumor-Intrinsic PIK3CA represses tumor immunogenecity in a model of pancreatic cancer. $J$ Clin Invest 2019;129:3264-76.

131 Yamamoto K, Venida A, Yano J, et al. Autophagy promotes immune evasion of pancreatic cancer by degrading MHC-I. Nature 2020;581:100-5.

132 Batlle E, Clevers H. Cancer stem cells revisited. Nat Med 2017:23:1124-34

133 Shibata M, Hoque MO. Targeting cancer stem cells: a strategy for effective eradication of cancer. Cancers 2019;11. doi:10.3390/ cancers11050732. [Epub ahead of print: 2705 2019].

134 Sultan M, Coyle KM, Vidovic D, et al. Hide-and-seek: the interplay between cancer stem cells and the immune system. Carcinogenesis 2017;38:107-18.

135 Morrison BJ, Steel JC, Morris JC. Reduction of MHC-I expression limits T-lymphocyte-mediated killing of Cancer-initiating cells. BMC Cancer 2018;18:469.

136 Di Tomaso T, Mazzoleni S, Wang E, et al. Immunobiological characterization of cancer stem cells isolated from glioblastoma patients. Clin Cancer Res 2010;16:800-13.

137 Pietra G, Manzini C, Vitale M, et al. Natural killer cells kill human melanoma cells with characteristics of cancer stem cells. Int Immunol 2009;21:793-801.

138 Volonté A, Di Tomaso T, Spinelli M, et al. Cancer-initiating cells from colorectal cancer patients escape from T cell-mediated immunosurveillance in vitro through membrane-bound IL-4. J Immunol 2014;192:523-32.

139 Yang W, Li Y, Gao R, et al. Mhc class I dysfunction of glioma stem cells escapes from CTL-mediated immune response via activation of Wnt/ $\beta$-catenin signaling pathway. Oncogene 2020;39:1098-111.

140 Tseng $\mathrm{H}-\mathrm{C}$, Arasteh A, Paranjpe A, et al. Increased lysis of stem cells but not their differentiated cells by natural killer cells; dedifferentiation or reprogramming activates NK cells. PLOS One 2010;5:e11590.

141 Castriconi R, Daga A, Dondero A, et al. Nk cells recognize and kill human glioblastoma cells with stem cell-like properties. J Immunol 2009;182:3530-9.

142 Costanzo V, Bardelli A, Siena S, et al. Exploring the links between cancer and placenta development. Open Biol 2018;8:180081.

143 Bossard C, Bézieau S, Matysiak-Budnik T, et al. HLA-E/B2 microglobulin overexpression in colorectal cancer is associated with recruitment of inhibitory immune cells and tumor progression. Int $J$ Cancer 2012;131:855-63.

144 Guo Z-Y, Lv Y-G, Wang L, et al. Predictive value of HLA-G and HLA-E in the prognosis of colorectal cancer patients. Cell Immunol 2015;293:10-16.

145 de Kruijf EM, Sajet A, van Nes JG, et al. Hla-E and HLA-G expression in classical HLA class I-negative tumors is of prognostic value for clinical outcome of early breast cancer patients. J Immunol 2010;185:7452-9.

146 Gooden M, Lampen M, Jordanova ES, et al. Hla-E expression by gynecological cancers restrains tumor-infiltrating $\mathrm{CD} 8^{+} \mathrm{T}$ lymphocytes. Proc Natl Acad Sci U S A 2011;108:10656-61. 
147 Ottenhoff THM, Joosten SA. Mobilizing unconventional T cells. Science 2019;366:302-3.

148 Joosten SA, Sullivan LC, Ottenhoff THM. Characteristics of HLA-E restricted T-cell responses and their role in infectious diseases. $J$ Immunol Res 2016;2016:1-11.

149 Benevolo M, Mottolese M, Tremante E, et al. High expression of HLA-E in colorectal carcinoma is associated with a favorable prognosis. J Transl Med 2011;9:184.

150 Djurisic S, Hviid TVF. Hla class Ib molecules and immune cells in pregnancy and preeclampsia. Front Immunol 2014;5:652.

151 Lee N, Ishitani A, Geraghty DE. Hla-F is a surface marker on activated lymphocytes. Eur J Immunol 2010;40:2308-18.

152 Lepin EJ, Bastin JM, Allan DS, et al. Functional characterization of HLA-F and binding of HLA-F tetramers to ILT2 and ILT4 receptors. Eur J Immunol 2000;30:3552-61.

153 Lin A, Yan W-H. The emerging roles of human leukocyte Antigen-F in immune modulation and viral infection. Front Immunol 2019;10:964.

154 Dulberger CL, McMurtrey CP, Hölzemer A, et al. Human leukocyte antigen $\mathrm{F}$ presents peptides and regulates immunity through interactions with NK cell receptors. Immunity 2017;46:1018-29. e1017.

155 Wu B, Yang H, Ying S, et al. High HLA-F expression is a poor prognosis factor in patients with nasopharyngeal carcinoma. Analytical Cellular Pathology 2018;2018:1-8.

156 Wuerfel FM, Huebner H, Häberle L, et al. Hla-G and HLA-F protein isoform expression in breast cancer patients receiving neoadjuvant treatment. Sci Rep 2020;10:15750.

157 Ishigami S, Arigami T, Okumura $\mathrm{H}$, et al. Human leukocyte antigen (HLA)-E and HLA-F expression in gastric cancer. Anticancer Res 2015;35:2279-85.

158 Lin A, Zhang X, Ruan Y-Y, et al. Hla-F expression is a prognostic factor in patients with non-small-cell lung cancer. Lung Cancer 2011;74:504-9.

159 Harada A, Ishigami S, Kijima Y, et al. Clinical implication of human leukocyte antigen (HLA)-F expression in breast cancer. Pathol Int 2015;65:569-74.

160 Zhang X, Lin A, Zhang J-G, et al. Alteration of HLA-F and HLA I antigen expression in the tumor is associated with survival in patients with esophageal squamous cell carcinoma. Int $J$ Cancer 2013;132:82-9.

161 Carosella ED, Moreau P, Lemaoult J, et al. Hla-G: from biology to clinical benefits. Trends Immunol 2008;29:125-32.

162 Tilburgs T, Evans JH, Crespo Ângela C, et al. The HLA-G cycle provides for both NK tolerance and immunity at the maternal-fetal interface. Proc Natl Acad Sci U S A 2015;112:13312-7.

163 Loustau M, Anna F, Dréan R, et al. Hla-G Neo-Expression on tumors. Front Immunol 2020;11:1685.

164 Yaghi L, Poras I, Simoes RT, et al. Hypoxia inducible factor-1 mediates the expression of the immune checkpoint HLA-G in glioma cells through hypoxia response element located in exon 2. Oncotarget 2016;7:63690-707.

165 Yie S-M, Yang H, Ye S-R, et al. Expression of HLA-G is associated with prognosis in esophageal squamous cell carcinoma. Am J Clin Pathol 2007;128:1002-9.

166 Zhang R-L, Zhang X, Dong S-S, et al. Predictive value of different proportion of lesion HLA-G expression in colorectal cancer. Oncotarget 2017;8:107441-51.

167 Wang Y, Fan X, Li H, et al. Tumor border sharpness correlates with HLA-G expression in low-grade gliomas. J Neuroimmunol 2015;282:1-6.

$168 \mathrm{He}$ X, Dong D-dan, Yie S-mian, et al. Hla-G expression in human breast cancer: implications for diagnosis and prognosis, and effect on allocytotoxic lymphocyte response after hormone treatment in vitro. Ann Surg Oncol 2010;17:1459-69.

169 Yie S-M, Yang H, Ye S-R, et al. Expression of human leukocyte antigen $\mathrm{G}$ (HLA-G) correlates with poor prognosis in gastric carcinoma. Ann Surg Oncol 2007;14:2721-9.

170 Ibrahim EC, Aractingi S, Allory Y, et al. Analysis of HLA antigen expression in benign and malignant melanocytic lesions reveals that upregulation of HLA-G expression correlates with malignant transformation, high inflammatory infiltration and HLA-A1 genotype. Int J Cancer 2004:108:243-50.

171 Yie S-mian, Yang $\mathrm{H}$, Ye S-rong, et al. Expression of human leucocyte antigen G (HLA-G) is associated with prognosis in nonsmall cell lung cancer. Lung Cancer 2007;58:267-74.

172 Jung YW, Kim YT, Kim SW, et al. Correlation of human leukocyte antigen-G (HLA-G) expression and disease progression in epithelia ovarian cancer. Reprod Sci 2009;16:1103-11.

173 Krijgsman D, Roelands J, Hendrickx W, et al. Hla-G: a new immune checkpoint in cancer? Int J Mol Sci 2020;21:4528.
174 Seliger B. Role of microRNAs on HLA-G expression in human tumors. Hum Immunol 2016:77:760-3.

175 Bian X, Si Y, Zhang M, et al. Down-expression of miR-152 lead to impaired anti-tumor effect of NK via upregulation of HLA-G. Tumor Biol 2016;37:3749-56.

176 Singer G, Rebmann V, Chen Y-C, et al. Hla-G is a potential tumor marker in malignant ascites. Clin Cancer Res 2003;9:4460-4.

177 Sun J, Chang Y-X, Niu C-Y. Evaluation of ascitic soluble human leukocyte antigen-G for distinguishing malignant ascites from benign ascites. Tumor Biology 2017;39:101042831772684.

178 Bassani-Sternberg M, Barnea E, Beer I, et al. Soluble plasma HLA peptidome as a potential source for cancer biomarkers. Proc Nat Acad Sci U S A 2010;107:18769-76.

179 Li J-B, Ruan Y-Y, Hu B, et al. Importance of the plasma soluble HLA-G levels for prognostic stratification with traditional prognosticators in colorectal cancer. Oncotarget 2017;8:48854-62.

180 Rouas-Freiss N, LeMaoult J, Verine J, et al. Intratumor heterogeneity of immune checkpoints in primary renal cell cancer: focus on HLA-G/ILT2/ILT4. Oncoimmunology 2017;6:e1342023.

181 Carosella ED, Rouas-Freiss N, Tronik-Le Roux D, et al. Hla-G: an immune checkpoint molecule. Adv Immunol 2015;127:33-144.

182 Dumont C, Jacquier A, Verine J, et al. CD8 ${ }^{+} \mathrm{PD}^{-1} 1^{-} \mathrm{ILT} 2^{+} \mathrm{T}$ Cells Are an Intratumoral Cytotoxic Population Selectively Inhibited by the Immune-Checkpoint HLA'G. Cancer Immunol Res 2019;7:1619-32.

183 García M, Palma MB, Verine J, et al. The immune-checkpoint HLA-G/ILT4 is involved in the regulation of VEGF expression in clear cell renal cell carcinoma. BMC Cancer 2020;20:624.

184 Böhm W, Thoma S, Leithäuser F, et al. T cell-mediated, IFNgamma-facilitated rejection of murine B16 melanomas. J Immunol 1998;161:897-908.

185 Dezfouli S, Hatzinisiriou I, Ralph SJ. Enhancing CTL responses to melanoma cell vaccines in vivo : synergistic increases obtained using IFN $\gamma$ primed and IFN $\beta$ treated B7-1 ${ }^{+} \mathrm{B} 16-\mathrm{F} 10$ melanoma cells. Immunol Cell Biol 2003;81:459-71.

186 Corrales L, Glickman LH, McWhirter SM, et al. Direct activation of sting in the tumor microenvironment leads to potent and systemic tumor regression and immunity. Cell Rep 2015;11:1018-30.

187 Demaria O, De Gassart A, Coso S, et al. Sting activation of tumor endothelial cells initiates spontaneous and therapeutic antitumor immunity. Proc Natl Acad Sci U S A 2015;112:15408-13.

188 Liu Y, Crowe WN, Wang L, et al. An inhalable nanoparticulate sting agonist synergizes with radiotherapy to confer long-term control of lung metastases. Nat Commun 2019;10:5108.

189 Mullins SR, Vasilakos JP, Deschler K, et al. Intratumoral immunotherapy with TLR7/8 agonist MEDI9197 modulates the tumor microenvironment leading to enhanced activity when combined with other immunotherapies. J Immunother Cancer 2019;7:244.

$190 \mathrm{Fu}$ J, Kanne DB, Leong M, et al. Sting agonist formulated cancer vaccines can cure established tumors resistant to PD-1 blockade. Sci Transl Med 2015;7:283ra52.

191 Koske I, Rössler A, Pipperger L, et al. Oncolytic virotherapy enhances the efficacy of a cancer vaccine by modulating the tumor microenvironment. Int J Cancer 2019;145:1958-69.

192 Vijayakumar G, McCroskery S, Palese P. Engineering Newcastle disease virus as an oncolytic vector for intratumoral delivery of immune checkpoint inhibitors and immunocytokines. $J$ Virol 2020;94. doi:10.1128/JVI.01677-19. [Epub ahead of print: 1701 2020].

193 Schrörs B, Boegel S, Albrecht C, et al. Multi-Omics characterization of the 4T1 murine mammary gland tumor model. Front Oncol 2020;10:1195.

194 Dang N, Waer M, Sprangers B, et al. Improved anti-tumour adaptive immunity can overcome the melanoma immunosuppressive tumour microenvironment. Cancers 2019;11:1694.

195 Chiang EY, Henson M, Stroynowski I. Correction of defects responsible for impaired qa-2 class lb MHC expression on melanoma cells protects mice from tumor growth. J Immunol 2003;170:4515-23.

196 Comiskey M, Goldstein CY, De Fazio SR, et al. Evidence that HLA-G is the functional homolog of mouse qa-2, the Ped gene product. Hum Immunol 2003;64:999-1004.

197 Joly E, Rouillon V. The orthology of HLA-E and H2-Qa1 is hidden by their concerted evolution with other MHC class I molecules. Biol Direct 2006;1:2.

198 Oliveira CC, van Veelen PA, Querido B, et al. The nonpolymorphic MHC Qa-1b mediates CD8+ T cell surveillance of antigenprocessing defects. J Exp Med 2010;207:207-21.

199 Haslam A, Prasad V. Estimation of the percentage of US patients with cancer who are eligible for and respond to checkpoint inhibitor immunotherapy drugs. JAMA Netw Open 2019;2:e192535. 
200 Strickler JH, Hanks BA, Khasraw M. Tumor mutational burden as a predictor of immunotherapy response: is more always better? Clin Cancer Res 2021;27:1236-41.

201 Ochoa de Olza M, Navarro Rodrigo B, Zimmermann S, et al. Turning up the heat on non-immunoreactive tumours: opportunities for clinical development. Lancet Oncol 2020;21:e419-30.

202 Yang RK, Qing Y, Jelloul FZ, et al. Identification of biomarkers of immune checkpoint blockade efficacy in recurrent or refractory solid tumor malignancies. Oncotarget 2020;11:600-18.

203 Klempner SJ, Fabrizio D, Bane S, et al. Tumor mutational burden as a predictive biomarker for response to immune checkpoint inhibitors: a review of current evidence. Oncologist 2020;25:e147-59.

204 Chowell D, Morris LGT, Grigg CM, et al. Patient HLA class I genotype influences cancer response to checkpoint blockade immunotherapy. Science 2018;359:582-7.

205 Shim JH, Kim HS, Cha H, et al. HLA-corrected tumor mutation burden and homologous recombination deficiency for the prediction of response to PD-(L)1 blockade in advanced non-small-cell lung cancer patients. Ann Oncol 2020;31:902-11.

206 Anagnostou V, Niknafs N, Marrone K, et al. Multimodal genomic features predict outcome of immune checkpoint blockade in nonsmall-cell lung cancer. Nat Cancer 2020;1:99-111.

207 Hiraoka N, Ino Y, Hori S, et al. Expression of classical human leukocyte antigen class I antigens, HLA-E and HLA-G, is adversely prognostic in pancreatic cancer patients. Cancer Sci 2020;111:3057-70

208 Imai D, Yoshizumi T, Okano S, et al. The prognostic impact of programmed cell death ligand 1 and human leukocyte antigen class I in pancreatic cancer. Cancer Med 2017;6:1614-26.

209 Kwak Y, Koh J, Park Y, et al. Differential prognostic impact of CD8+ T cells based on human leucocyte antigen I and PD-L1 expression in microsatellite-unstable gastric cancer. $\mathrm{Br} \mathrm{J}$ Cancer 2020;122:1399-408.

210 Park Y, Koh J, Kwak Y, et al. Clinicopathologic significance of human leukocyte antigen class I expression in patients with stage II and III gastric cancer. Cancer Immunol Immunother 2019;68:1779-90

211 Mizukami Y, Kono K, Maruyama T, et al. Downregulation of HLA class I molecules in the tumour is associated with a poor prognosis in patients with oesophageal squamous cell carcinoma. $\mathrm{Br} J$ Cancer 2008:99:1462-7.

212 Ito S, Okano S, Morita M, et al. Expression of PD-L1 and HLA class I in esophageal squamous cell carcinoma: prognostic factors for patient outcome. Ann Surg Oncol 2016;23:508-15.
213 Cabrera T, López-Nevot M, Gaforio J, et al. Analysis of HLA expression in human tumor tissues. Cancer Immunol Immunother 2003;52:1-9.

214 Tremante E, Lo Monaco E, Ingegnere T, et al. Monoclonal antibodies to HLA-E bind epitopes carried by unfolded $\beta_{2} \mathrm{~m}$-free heavy chains. Eur J Immunol 2015;45:2356-64.

215 Rosenbaum E, Seier K, Bandlamudi C, et al. Hla genotyping in synovial sarcoma: identifying HLA-A*02 and its association with clinical outcome. Clin Cancer Res 2020;26:5448-55.

216 Borst L, van der Burg SH, van Hall T. The NKG2A-HLA-E axis as a novel checkpoint in the tumor microenvironment. Clin Cancer Res 2020;26:5549-56.

217 Perea F, Sánchez-Palencia A, Gómez-Morales M, et al. Hla class I loss and PD-L1 expression in lung cancer: impact on T-cell infiltration and immune escape. Oncotarget 2018;9:4120-33.

218 Rodig SJ, Gusenleitner D, Jackson DG, et al. Mhc proteins confer differential sensitivity to CTLA-4 and PD-1 blockade in untreated metastatic melanoma. Sci Trans/ Med 2018;10:eaar3342.

219 Yoo SH, Keam B, Ock C-Y, et al. Prognostic value of the association between MHC class I downregulation and PD-L1 upregulation in head and neck squamous cell carcinoma patients. Sci Rep 2019;9:7680.

220 Ozcan M, Janikovits J, von Knebel Doeberitz M, et al. Complex pattern of immune evasion in MSI colorectal cancer. Oncoimmunology 2018;7:e1445453.

221 Na HY, Park Y, Nam SK, et al. Expression of human leukocyte antigen class I and $\beta 2$-microglobulin in colorectal cancer and its prognostic impact. Cancer Sci 2021;112:91-100.

222 Umemoto Y, Okano S, Matsumoto Y, et al. Prognostic impact of programmed cell death 1 ligand 1 expression in human leukocyte antigen class I-positive hepatocellular carcinoma after curative hepatectomy. J Gastroenterol 2015;50:65-75.

223 Tsang JY, Ho C-S, Ni Y-B, et al. Co-Expression of HLA-I loci improved prognostication in HER2+ breast cancers. Cancer Immunol Immunother 2020;69:799-811.

224 Sinn BV, Weber KE, Schmitt WD, et al. Human leucocyte antigen class I in hormone receptor-positive, HER2-negative breast cancer: association with response and survival after neoadjuvant chemotherapy. Breast Cancer Res 2019;21:142.

225 Ferns DM, Heeren AM, Samuels S, et al. Classical and nonclassical HLA class I aberrations in primary cervical squamousand adenocarcinomas and paired lymph node metastases. $J$ Immunother Cancer 2016:4:78. 